\title{
Rapid reconstruction of neural circuits using tissue expansion and lattice light sheet microscopy
}

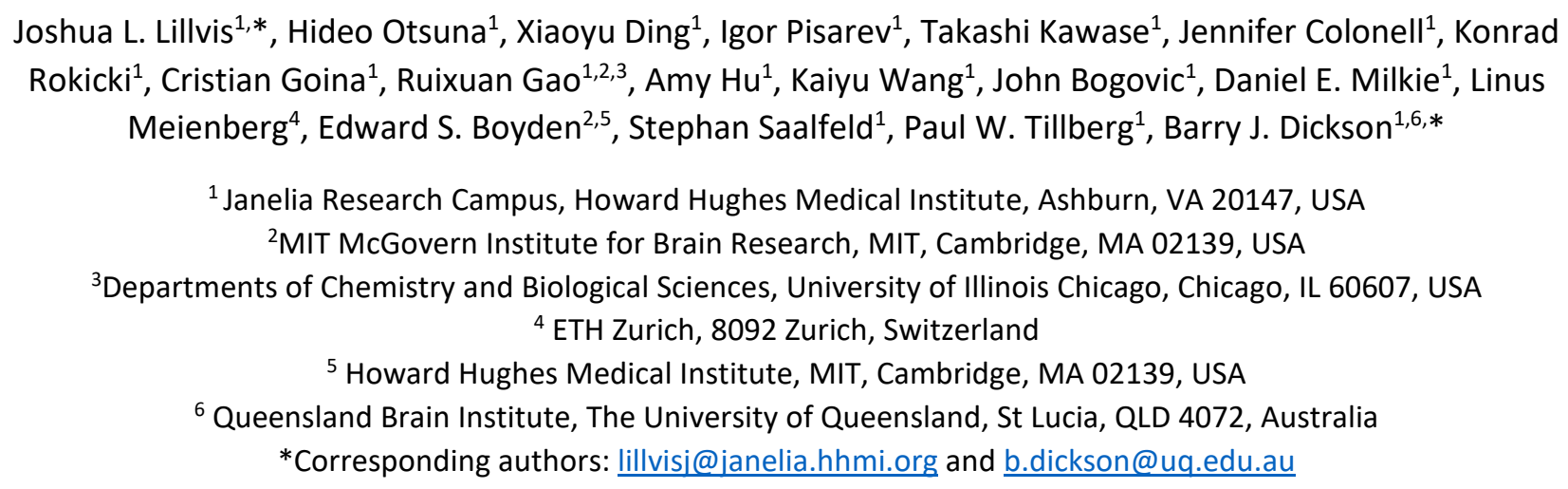

\begin{abstract}
Electron microscopy (EM) allows for the reconstruction of dense neuronal connectomes but suffers from low throughput, limiting its application to small numbers of reference specimens. We developed a protocol and analysis pipeline using tissue expansion and lattice light-sheet microscopy (ExLLSM) to rapidly reconstruct selected circuits across many samples with single synapse resolution and molecular contrast. We validate this approach in Drosophila, demonstrating that it yields synaptic counts similar to those obtained by EM, can be used to compare counts across sex and experience, and to correlate structural connectivity with functional connectivity. This approach fills a critical methodological gap in studying variability in the structure and function of neural circuits across individuals within and between species.
\end{abstract}

Main

\section{Introduction}

Major efforts are underway to reconstruct comprehensive wiring diagrams of the nervous systems of various species. These connectome projects are fueled by recent advances in electron microscopy and automated image analysis and motivated by the idea that knowing the exact pattern of synaptic connectivity within a neural network is necessary, though not sufficient, to understand how it functions. Currently, most connectome projects are focused on generating reference connectivity maps for selected model organisms (White et al., 1986; Ryan et al., 2016; Eichler et al., 2017; Hildebrand et al., 2017; Zheng et al., 2018; Cook et al., 2019; Scheffer et al., 2020; Consortium et al., 2021). This current state of connectomics research is somewhat analogous to the state of genomics research just over two decades ago. Advances in DNA sequencing had made it possible to generate reference genomes for selected model organisms, and ultimately also for humans. The success of these genome projects created the need to rapidly resequence targeted genomic regions across large numbers of samples in order to determine how these sequences vary from individual to individual and from species to species, so as to better understand how the genome "works" and how it evolves. Similarly, with the expanding collection of reference connectomes, there is now an increasingly urgent need for methods that allow rapid but sparse reconstruction of neural circuits across large numbers of samples. 
Electron microscopy (EM), the method of choice for the reconstruction of dense reference connectomes, is not well-suited to this task. The time and cost involved in EM reconstruction currently limit such comparative analyses to very small volumes (Bourne and Harris, 2012; Bumbarger et al., 2013; Valdes-Aleman et al., 2021) and to a few well-equipped laboratories. Moreover, because EM produces a dense image without molecular specificity, it is exceedingly difficult to highlight individual neurons and molecules. EM thus provides both too much and too little information for a typical comparative analysis of neuronal connectivity: too much, in that the specific neurons of interest first need to be identified amongst the vast tangle of processes revealed in the EM images, and too little, in that essential information on, for example, the chemical nature of individual connections, is generally not available. Light microscopy provides the means to readily highlight the neurons and molecules of interest across many individual animals but has traditionally lacked the resolution needed to resolve individual synaptic connections. Recently, however, a combination of expansion microscopy (Tillberg et al., 2016) and lattice light sheet imaging (Chen et al., 2014a) (ExLLSM) has been shown to afford sufficient resolution to reveal single synapses and molecular labels and to have sufficient speed to image large volumes of neural tissue across many animals (Gao et al., 2019).

Here, we report the development of an ExLLSM method and pipeline to rapidly resample selected circuits within the Drosophila melanogaster connectome. We show that synaptic counts obtained by this method are consistent with those obtained by EM, and that it can reveal structures such as electrical connections that are largely invisible to EM. Moreover, we demonstrate the application of our ExLLSM pipeline in comparative approaches that reveal state-dependent differences in neuronal connectivity, and correlate structural, physiological, and behavioral data across multiple individuals.

\section{Data acquisition and analysis}

We introduced an interpenetrating network gel to increase specimen expansion (from $4 \mathrm{X}$ to $8 \mathrm{X}$ ), while also improving mechanical stability, allowing reliable multiple day imaging without sample shrinking or further expansion. With the LLSM settings used, the central brain of Drosophila can be imaged in three colors in $\sim 5$ days at a resolution $(\sim 30 \times 30 \times 100 \mathrm{~nm})$ sufficient to identify individual electrical and chemical presynaptic and postsynaptic sites across the brain.

To process and analyze the images obtained by 8X ExLLSM (hereafter referred to as ExLLSM), we developed a largely automated pipeline to quantify synaptic connectivity between genetically identified neurons (Fig. 1A). The pipeline consists of a previously published automated image tile processing and stitching method, consisting of flat field correction, deconvolution, and tile stitching (Gao et al., 2019); software to visualize in 3D and to semiautomatically segment multi-terabyte light microscopy image volumes (Figure 1B-G, Sup. Fig. 1); automated neuron segmentation (Sup. Fig. 3) and synapse classifier workflows (Figure 1L-Q); and an automated workflow to segment individual pre and postsynaptic sites, assign them to segmented neuron masks, and identify connections (Sup. Fig. 2). Finally, our pipeline quantifies the connections, sizes, and locations of the synaptic sites and exports the results as images and tables.

Developing this pipeline required updates to the VVD Viewer light microscopy visualization and analysis software (Wan et al., 2012) to allow smooth 3D visualization and segmentation of multi-terabyte datasets. The VVD software can be utilized to segment neurons, check and refine automatic segmentation results, generate groundtruth data for automatic segmentation network training, and create high quality videos. In addition, we have developed several Fiji plugins (Schindelin et al., 2012) and Apache Spark-based tools (Zaharia et al., 2016) to simplify and accelerate the processing and analyzing of big image data. These include cropping, MIP creation, pixel 
A

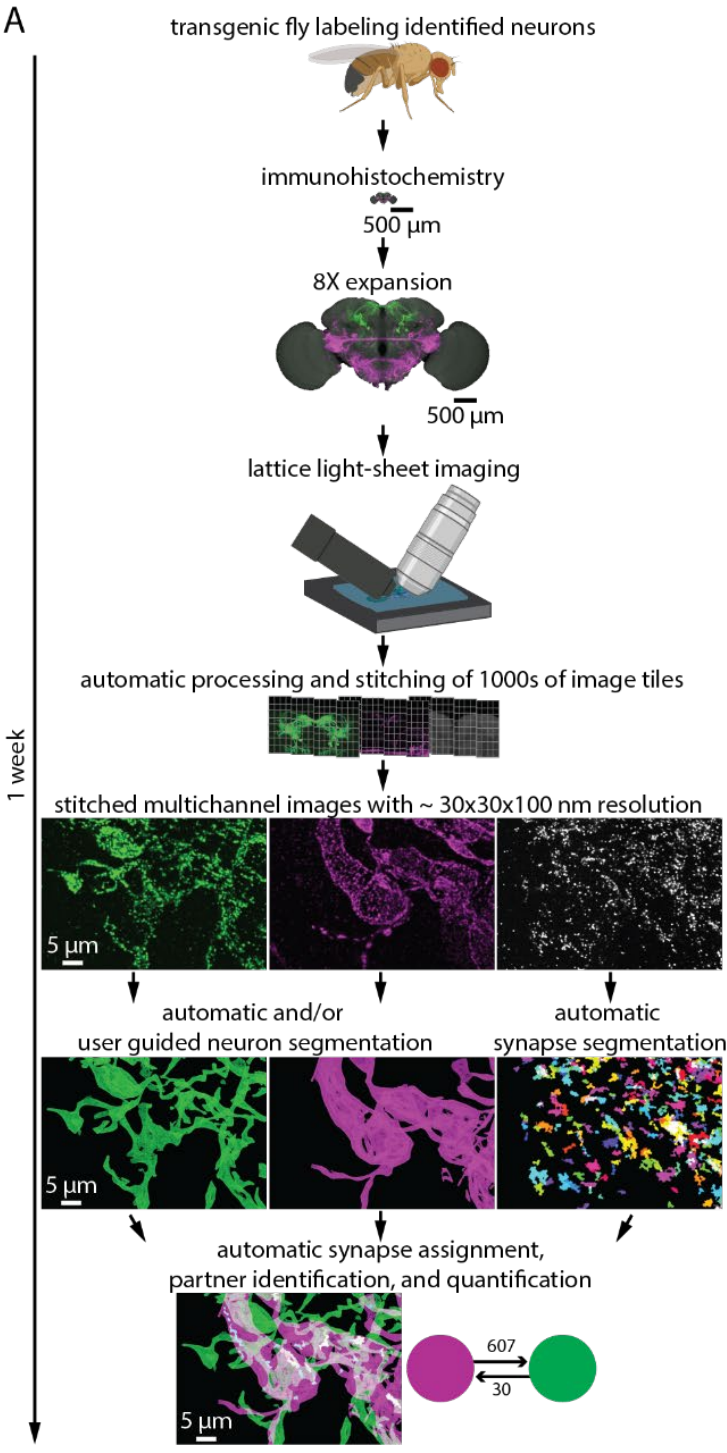

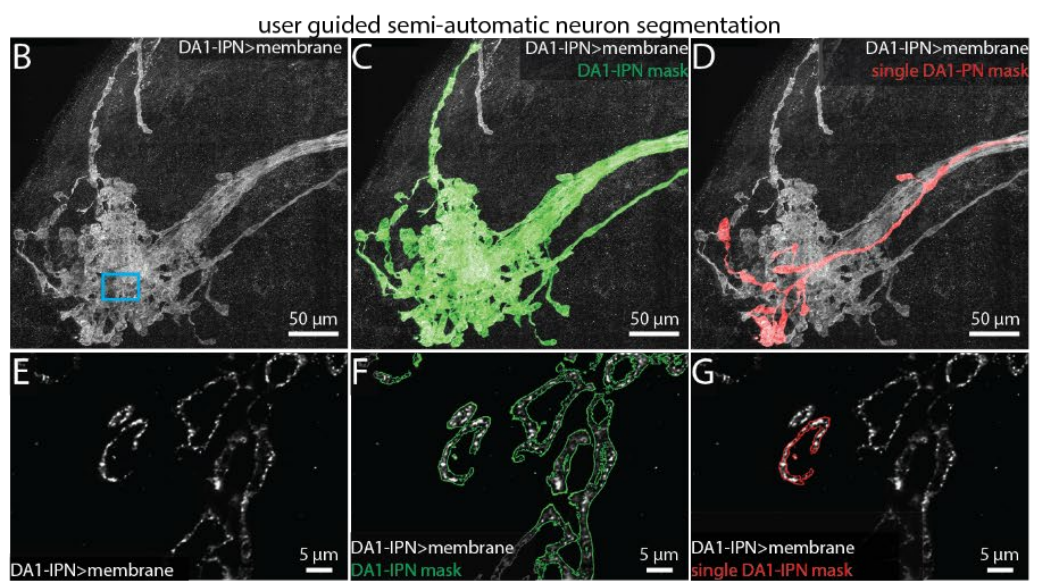
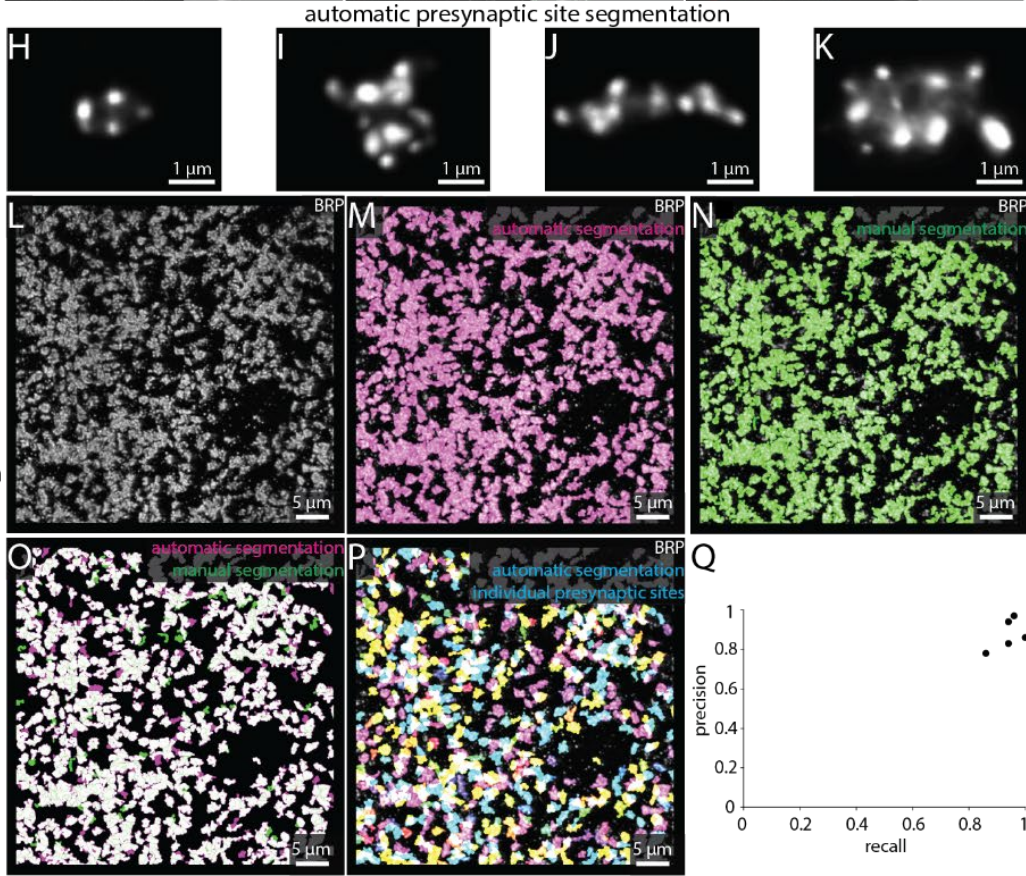

$\mathrm{Q}$

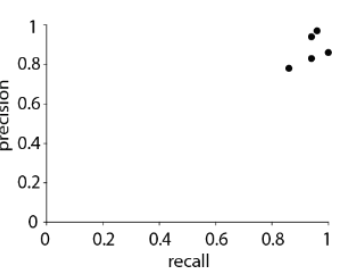

Figure 1 Largely automated ExLLSM image processing and analysis pipeline. (A) Overview. (B-G) Semi-automatic neuron segmentation. DA1IPN neuron cluster (B, white) in the lateral horn with neuron masks generated by semi-automatic segmentation of the entire cluster (green, $C)$ and manual segmentation of a single neuron (red, D). (E-G) Single z-slice of boxed region in (B-D) showing a cross-section of presynaptic boutons. (H-Q) Automatic presynaptic site segmentation. (H-K) Examples of individual presynaptic site morphologies at $8 X$ as visualized by labeling BRP. Crop from the optic lobe showing the BRP label (white, L) with automatically (magenta, M) and manually (ground truth, green, $N)$ segmented presynaptic sites. (O) Overlay of automatic presynaptic site segmentation results (magenta) and ground truth data (green). $(P)$ Overlay of BRP (white) and automatically segmented individual presynaptic sites (multicolor). (Q) Precision-recall plot of automatic presynaptic site segmentation. Results from 5 independent samples and 5 different brain regions.

intensity thresholding, signal cross-talk subtraction, pixel shape transformation, 3D component connecting, component analysis, and component size thresholding.

We assembled these open source tools into easy-to-use computational workflows via Nextflow (Di Tommaso et al., 2017) (Sup. Fig. 1-3). All of the tools are described in the methods and on GitHub (https://github.com/JaneliaSciComp/exllsm-circuit-reconstruction) (Lillvis et al., 2021), where they are maintained along with user manuals and usage examples. The pipeline was built to analyze multi-terabyte $8 \mathrm{X}$ ExLLSM images of the Drosophila nervous system. However, the only aspects of the pipeline that are specific to this data are the trained convolutional neural network (Çiçek et al., 2016) models used for synapse detection and 
neuron segmentation (Fig. 1L-Q, Sup. Fig. 3). These models can be trained on new data from other organisms or microscopes and seamlessly integrated into the pipeline.

\section{Presynaptic site counts obtained by ExLLSM match those from EM}

To validate our data acquisition and analysis pipeline, we compared presynaptic site counts obtained via ExLLSM to EM. In Drosophila, presynaptic active zones (hereafter referred to as presynaptic sites) are anatomically identified in EM images by T-bars (Huang et al., 2018; Buhmann et al., 2021), where synaptic vesicles pool and are released. One component of T-bars is the Bruchpilot (Brp) protein (Vactor and Sigrist, 2017), which can be detected in LM images through either ubiquitous (Wagh et al., 2006) or genetically-restricted labeling (Chen et al., 2014b). We therefore used BRP as the marker to identify presynaptic sites of three distinct neuron types optic lobe L2 neurons, antennal lobe DA1-IPN neurons, and ascending SAG neurons - and compared presynaptic site counts obtained by ExLLSM to T-bar counts obtained via EM.

Optic lobe L2 neurons make synapses onto motion detecting neurons in the medulla (Takemura et al., 2013; Tuthill et al., 2013). In an EM volume comprising 7 optic lobe columns from one animal obtained by focused-ion beam milling scanning electron microscopy (FIB-SEM), an average of 207 presynaptic sites were detected per L2 neuron (Takemura et al., 2015). We used both the ubiquitous and genetically-restricted strategies to label presynaptic sites in L2. For ubiquitous labelling, we used the nc82 antibody to label BRP (Wagh et al., 2006) and a split-GAL4 driver line to specifically label the $L 2$ neurons (Tuthill et al., 2013) (Figure 2A-G, Video 1). We imaged large sections of the optic lobe medulla and segmented ten individual $L 2$ neurons in each of three flies and counted an average of 210 presynaptic sites per L2 neuron (Figure 2C). For genetically-restricted labelling, we used the synaptic tagging with recombination (STaR) method (Chen et al., 2014b) to specifically label the endogenous BRP protein in L2 neurons (Fig. $2 \mathrm{H}-\mathrm{J}$ ). With this method, we counted an average of 195 synapses, also across a total of $30 \mathrm{~L} 2$ neurons (Fig. 2C). Thus, the L2 synapse counts obtained by 8X ExLLSM were similar with both labeling methods and matched the EM counts (Fig. 2C).

The DA1-IPN neurons comprise a group of 7-8 sexually-dimorphic neurons that relay conspecific odors from the DA1 glomerulus in the antennal lobe to the mushroom body and lateral horn (Marin et al., 2002; Wong et al., 2002; Stockinger et al., 2005; Jefferis et al., 2007; Kurtovic et al., 2007; Kohl et al., 2013) (Figure 2K-P). We labeled these neurons using a LexAGAD driver line, imaged, segmented, and masked their projections in the lateral horn, and quantified presynaptic site counts in 11 females and 10 males (Fig. 2K-P). We compared these counts to those obtained from one hemisphere of the FIB-SEM female hemibrain (Scheffer et al., 2020) and both hemispheres in the TEM volume of another female (Zheng et al., 2018; Bates et al., 2020). On average, the female ExLLSM presynaptic site count of 2251 was similar to both EM datasets (FIB-SEM hemibrain: 2274; TEM: 1837 left, 1735 right) (Figure 2M). Although the average count in males, 2466, was higher than in females, this difference was not statistically significant (t-test, two-tailed $\mathrm{P}=0.25$ ).

The bilaterally-paired female-specific SAG ascending neurons relay mating status of the female fly to the central brain (Feng et al., 2014). In 18 females, we labeled SAG via a LexAGAD driver line, segmented the SAG neurons from the off-target neurons, and counted an average of 1440 presynaptic sites (Fig. 2Q-S, Video 2-3). In the FIBSEM female hemibrain (Scheffer et al., 2020), the SAG neurons have 1198 presynaptic sites, which falls within the range of counts we obtained by ExLLSM (Figure 2S). 

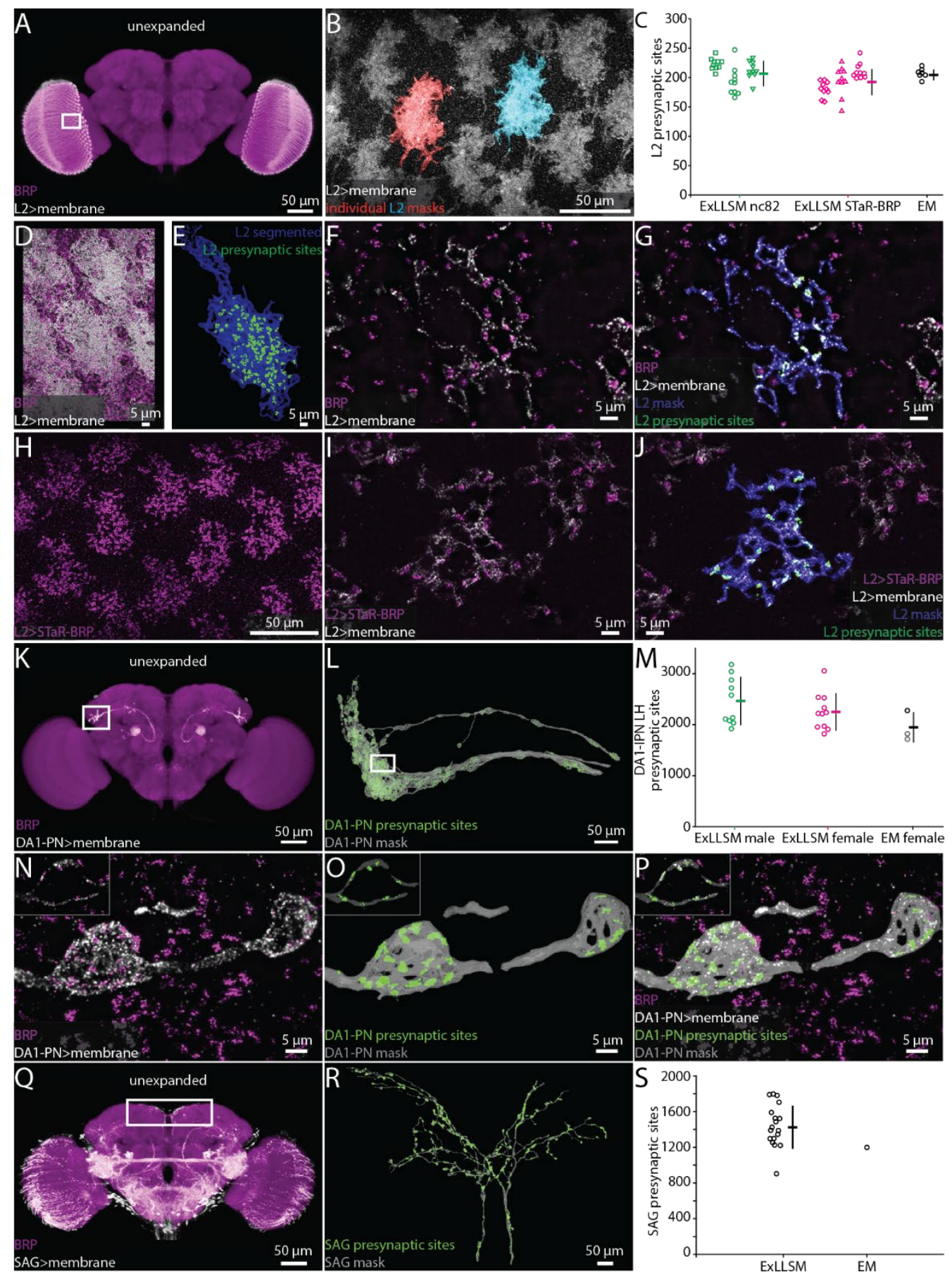

Figure 2 Automatic presynaptic site segmentation and neuron assignment. (A-B, D-G) Representative examples of quantifying presynaptic sites in $L 2$ neurons via ubiquitous BRP labeling. (A) Unexpanded female brain labeled to reveal BRP (magenta) and the membranes of $L 2$ neurons (white). (B) Representative $8 X$ view of $L 2$ neurons from rectangle region in (A), with two individual L2 neurons segmented. (C) Quantification of L2 presynaptic sites detected by ExLLSM using either ubiquitous (nc82) or restricted (STaR-BRP) labelling ( $n=10$ neurons, in each of 3 samples for both) and by the presence of T-bars in EM ( $n=7$ neurons in adjacent columns). (D) Group of $L 2$ neurons and BRP. (E) Mask of single segmented $L 2$ neuron from (D) with automatically identified $L 2$ presynaptic sites. (F) Zoom in on $5 z$-slices from (D) with analyses overlaid (G). (H-J) Representative example of quantifying presynaptic sites in L2 via STaR-BRP labeling. (G) STaR-BRP expression in a group of L2 neurons. BRP is only labeled in $L 2$ neurons. (I) Zoom in on 5 z-slices from $H$ with individual L2 mask and automatically identified L2 
presynaptic sites overlaid (J). (K-L, N-P) Representative example of quantifying presynaptic sites in DA1-IPN neurons via ubiquitous BRP labeling by the nc82 antibody. (K) Unexpanded view of female brain with BRP and the membrane of DA1-IPN neurons labeled. (L) $8 X$ view of segmented DA1-IPN neurons in the lateral horn, approximately from rectangle region in (J) with automatically detected DA1-IPN presynaptic sites labeled. (M) Quantification of DA1-IPN lateral horn presynaptic sites. Neuron cluster grouped across 11 female and 10 male ExLLSM samples, and 2 EM samples (FIB-SEM in black, left and right TEM in grey). (N) Zoom in on raw signal of two DA1-IPN presynaptic boutons from rectangle region in (L). Inset shows a single $z$-slice. (O) Analyzed data from N. (P) Overlay of raw data from $(N)$ and analyzed data from (O). ( $Q-R)$ Representative example of quantifying presynaptic sites in SAG neurons via ubiquitous BRP labeling by the nc82 antibody. Unexpanded view of a female brain with BRP and the membrane of SAG (and off-target) neurons labeled. (R) $8 X$ view of segmented SAG neurons from rectangle region in (Q) with automatically detected SAG presynaptic sites labeled. (S) Quantification of SAG presynaptic sites in 18 ExLLSM samples and 1 EM sample. (C, M, S) Individual samples and mean \pm SD are plotted.

\section{Chemical connectivity between identified neurons}

To test whether ExLLSM counts of chemical connections between identified neurons are also similar to counts obtained from EM, we focused on connections from the two cholinergic SAG neurons to their primary downstream targets, the ten pC1 neurons (Wang et al., 2020a) (Fig. 3A). Most Drosophila synapses are polyadic (Scheffer et al., 2020), such that a single presynaptic site will have multiple postsynaptic contacts. In the FIB-SEM hemibrain data set, the two SAG neurons make 5534 connections to downstream neurons from 1198 presynaptic sites. Of these connections, 938 are made to $\mathrm{pC} 1$ neurons via 677 SAG presynaptic sites.

Although ExLLSM is in principle compatible with any primary antibody, there is no known postsynaptic analog to BRP that labels all or most postsynaptic sites in Drosophila. Additionally, genetic, histochemistry, or imaging constraints make it difficult to specifically label distinct molecules in different neuron types in the same animal. In particular, our current ExLLSM protocol is limited to three-color imaging, which makes it challenging to simultaneously visualize two cell types and two molecular markers. To circumvent this problem, we tested whether we could quantify connectivity in two ways: 1) by labeling all presynaptic sites, the membrane of presynaptic neurons, and postsynaptic sites only in the postsynaptic neurons (Fig. 3B-F); and 2) by labeling all presynaptic sites and the membranes of both pre- and postsynaptic neurons (Fig. 31-O). The first approach allows us to quantify both the total number of SAG>pC1 connections ( 938 from EM) and the number of SAG presynaptic sites making these connections (677 from EM). The second approach only allows us to quantify the number of SAG presynaptic sites making connections to pC1 (677 from EM).

For both methods, we used the LexAGAD driver line to label the SAG neurons (Feng et al., 2014), a split-GAL4 line for the pC1 neurons (Wang et al., 2020a), and the BRP antibody to label all presynaptic sites (Figure 3A). In the first approach, we labeled putative cholinergic receptors in pC1 using a genetic reporter that labels Drep2 proteins in a neuron-specific manner. Although this reporter does not label endogenous Drep2 protein, it co-localizes with the acetylcholine receptor subunit D 7 at cholinergic synapses (Andlauer et al., 2014). Using this approach (Fig. $3 B-F$, Video 2), we counted an average of 729 SAG to $\mathrm{pC} 1$ connections made by an average of 583 presynaptic sites ( $n=11$ ) (Fig. 3G). Both numbers are slightly lower than the counts obtained from the FIB-SEM hemibrain (938 and 677, respectively), but we note that the counts for this EM sample fall within the range we obtained by ExLLSM (Fig. 3G).

Using the second approach to quantify connectivity, which quantifies the number of SAG presynaptic sites that contact the pC1 membrane (Fig. 3I-O, Video 3), we obtain an average of 501 connections (n=6) (Fig. 3G). The most connections we counted in a single sample was 604 , below the count of 677 in the EM sample, suggesting that this method may more consistently undercount synapses. Postsynaptic sites are often found at fine neural processes (Schneider-Mizell et al., 2016; Scheffer et al., 2020), and without specific labeling using a postsynaptic 

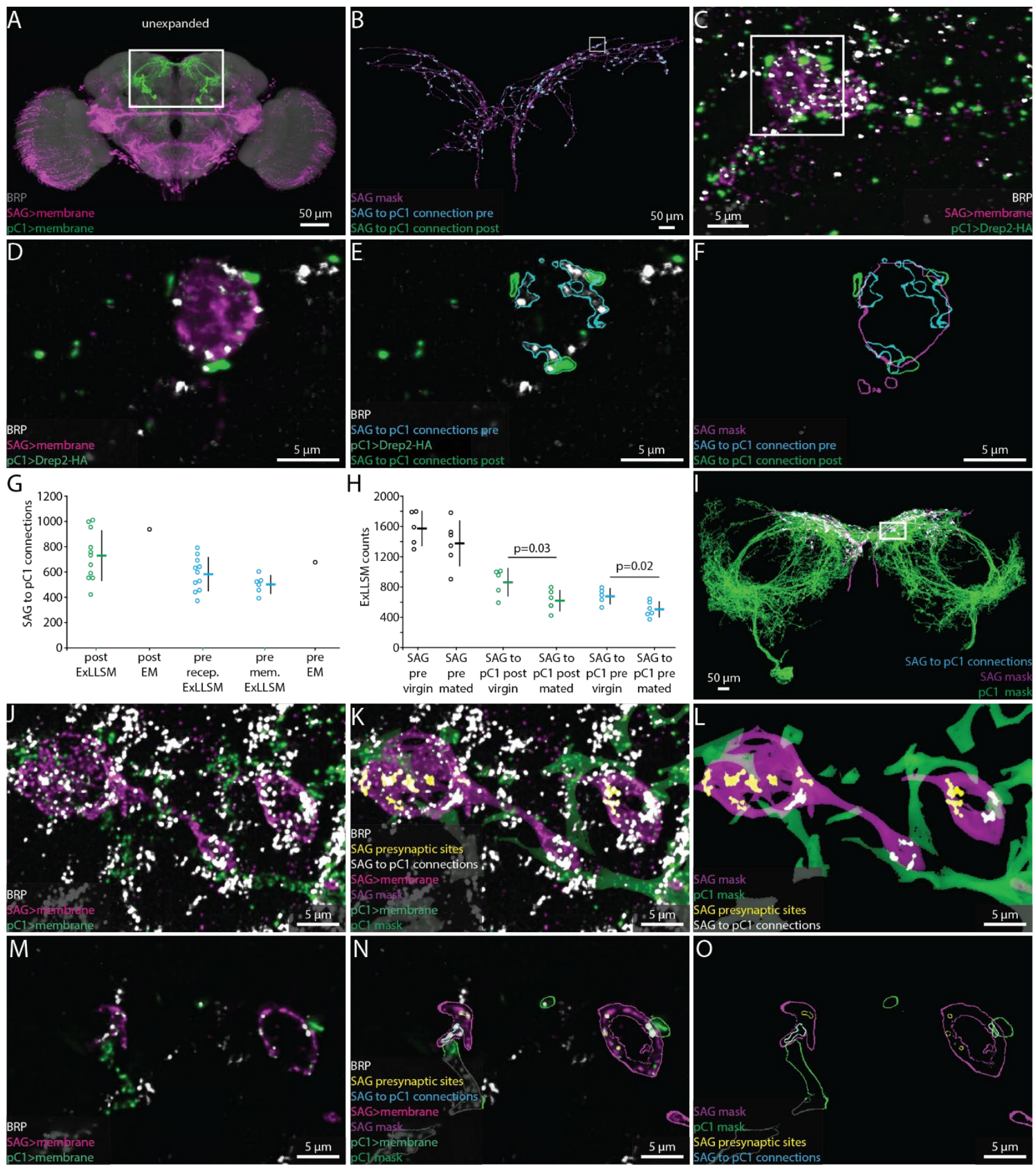

Figure 3 Quantifying connectivity using ExLLSM. (A) Unexpanded female brain labeled to reveal SAG (magenta) and pC1 (green) neurons and BRP presynaptic sites (nc82, grey). (B-F) Representative examples of quantifying connectivity via $p C 1$ postsynaptic site contact with SAG presynaptic sites. (B) Segmented SAG mask and the automatically detected pre- (cyan) and postsynaptic sites (green) of SAG to $p C 1$ connections. (C) max intensity projection of $200 \mathrm{z}$ slices from region approximately in the rectangle in (B). (D-F) Single z slice of region in the rectangle from (C). (G) Quantification of SAG to $p C 1$ connections. SAG to $p C 1$ connections as quantified by the number of $p C 1$ postsynaptic sites that contact SAG presynaptic sites in 11 ExLLSM samples (post ExLLSM) and the number of pC1 postsynaptic sites that connect to SAG presynaptic sites in 1 EM sample 
(connections, post EM). SAG presynaptic sites that make connections to $p C 1$ as quantified by the number of SAG presynaptic sites that contact $p C 1$ postsynaptic sites in 11 ExLLSM samples (pre recep. ExLLSM), number of SAG presynaptic sites that contact the pC1 membrane in 6 ExLLSM samples (pre mem. ExLLSM), and number of SAG presynaptic sites that make connections to $p C 1$ postsynaptic sites in 1 EM sample (pre EM). (H) Quantification of SAG presynaptic sites and SAG to $p C 1$ connections in virgin and mated female ExLLSM samples. Number of SAG presynaptic sites in 5 virgin samples (SAG pre virgin) and 6 mated samples (SAG pre mated). SAG to $p C 1$ connections as quantified by the number of $p C 1$ postsynaptic sites that contact SAG presynaptic sites in 5 virgin samples (SAG to $p C 1$ post virgin) and in 6 mated samples (SAG to pC1 post mated). $S A G$ presynaptic sites that make connections to $p C 1$ as quantified by the number of SAG presynaptic sites that contact $p C 1$ postsynaptic sites in 5 virgin samples (SAG to $p C 1$ pre virgin) and 6 mated samples (SAG to $p C 1$ pre mated). Virgin pre-and post-synaptic ExLLSM counts are significantly different from mated pre-and post-synaptic ExLLSM counts (t-tests). Individuals and mean $\pm S D$ plotted in $\mathrm{G}-\mathrm{H}$. (I-O) Quantifying connectivity via $p C 1$ membrane contact with SAG presynaptic sites. (I) Segmented masks of SAG and $p C 1$ and SAG to $p C 1$ connections approximately in rectangle in (A). (J-L) 100 z slices of region approximately in the rectangle in (I). (M-O) Single z slice of data from (J-L). Mask outlines shown.

marker such as Drep2, some of these fine processes may be missed due to the inherent limitations of the immunohistochemistry, $8 \mathrm{X}$ expansion, and the segmentation methods used.

We conclude that the ExLLSM reconstruction strategies used here result in similar connection counts that are in general agreement with the those obtained by EM. We recommend the use of a specific postsynaptic marker where practical to avoid undercounting. Nonetheless, we note that all of these approaches, including EM (Scheffer et al., 2020), rely on detection methods that generally have high specificity but lower sensitivity, and therefore all undercount connections to various degrees. In general, however, relative synaptic counts are more informative than absolute numbers. Because ExLLSM enables a much larger number of samples to be surveyed than EM, it should be able to provide a significantly more accurate assessment of relative connectivity regardless of which labeling strategy is used.

To assess the power of ExLLSM to reveal relative differences in synaptic connectivity, we tested the hypothesis that SAG neurons make more synaptic connections with pC1 neurons in virgin females than in mated females. The pC1 neurons regulate female receptivity and egg-laying (Wang et al., 2020a, 2020b), both of which change dramatically after mating. After mating, sensory neurons in the uterus detect the presence of a male seminal fluid protein (Häsemeyer et al., 2009; Yang et al., 2009), and the SAG neurons relay this signal from the uterus to pC1 neurons in the brain (Feng et al., 2014). Both the SAG and pC1 neurons have higher basal activity in virgin females than in mated females (Feng et al., 2014; Wang et al., 2020a), and so we hypothesized that they may also have more synaptic connections in virgin females than in mated females. We tested this hypothesis by counting SAG>pC1 synapses in ExLLSM brain samples from a total of 5 virgins and 6 mated females using the Drep2 labelling strategy. The average number of SAG presynaptic sites was similar in mated and virgin females (Fig. $3 \mathrm{H}$, $\mathrm{t}$-test, two-tailed $\mathrm{P}=0.26$ ) but $25 \%$ fewer of these presynaptic sites were connected to $\mathrm{pC} 1$ in mated females, resulting in $28 \%$ fewer connections (Fig. $3 \mathrm{H}$, t-test, two-tailed $\mathrm{P}=0.03$ and 0.02 , respectively). These data establish that SAG>pC1 synapses are indeed remodeled after mating and, moreover, demonstrate the power of ExLLSM to reveal and quantify state-dependent changes in neuronal connectivity.

\section{Detection and characterization of electrical connections}

Neurons also communicate through electrical connections called gap junctions (Güiza et al., 2018; Nagy et al., 2018). Gap junctions are difficult to detect in EM images. Invertebrate gap junction channels are formed by innexin proteins (Syrjanen et al., 2021), which provide a molecular label to visualize and quantify electrical connections using ExLLSM. Antibody staining against gap junction proteins reveals both distributed punctate signals and pronounced aggregations called plaques (Phelan et al., 2008; Markert et al., 2016). Although punctate signals may 


\section{Rapid reconstruction of neural circuits using tissue expansion and lattice light sheet microscopy}

label hemichannels in some instances, correlative superresolution light and electron microscopy in C. elegans has demonstrated that punctate signals can label gap junctions as well (Markert et al., 2016).

We used an antibody against innexin 6 (INX6) to assess the ability of our ExLLSM pipeline to detect and classify possible electrical connections. As previously reported (Wu et al., 2011), INX6 immunoreactivity was most pronounced in the fan-shaped body (Fig. 4A-D). In unexpanded tissue, fan-shaped body INX6 immunoreactivity resembles plaques (Fig. 4A). In expanded tissue, these plaques were revealed to be composed of clusters of INX6 (Fig. 4C). These INX6 clusters generally resembled the clusters of pre- and post-synaptic proteins found at chemical synapses (Fig. 1-3), and could be automatically classified as possible gap junctions using the same workflows used to detect chemical synapses (Fig. 4B-D).
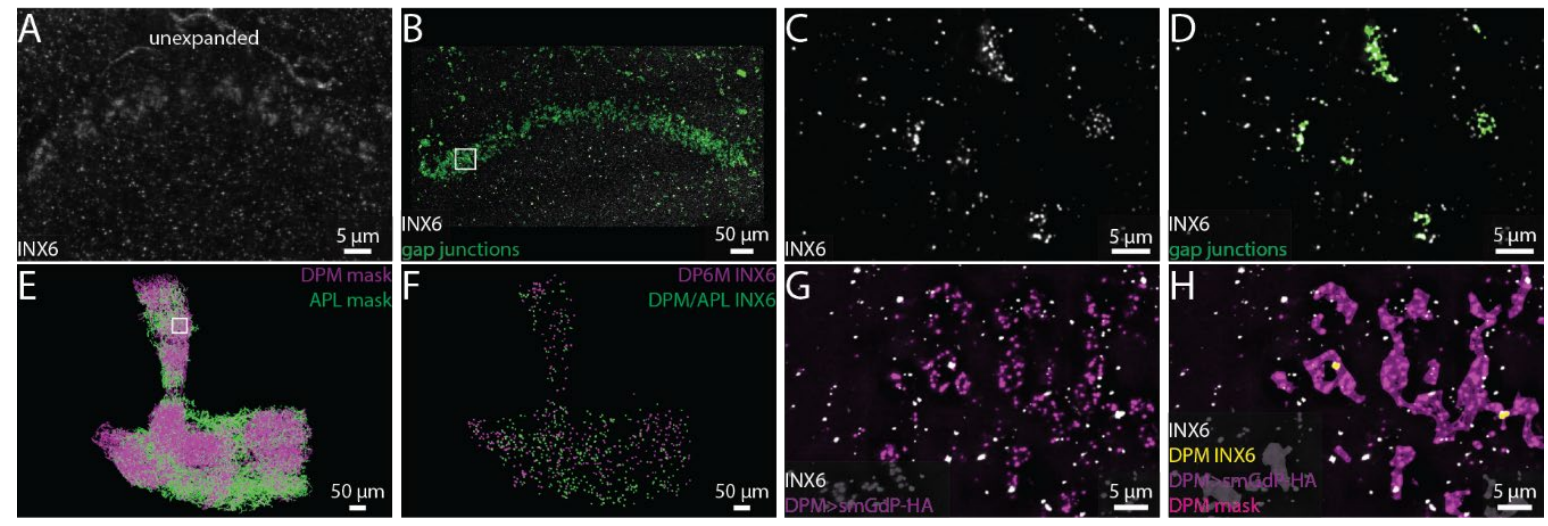

Figure 4 Visualizing gap junction proteins with ExLLSM. INX6 immunoreactivity in the fan shaped body in the unexpanded (A) and expanded (B) brain. (B) INX6 and automatically detected putative gap junctions (green). (C-D) Zoom in on region in rectangle in (B). (E) Segmented $D P M$ and APL neuron masks. (F) Automatically detected possible DPM and APL gap junction sites. (G-H) Zoom in on region in rectangle from (E) with INX6 (white), the DPM membrane (purple), DPM mask (magenta), and possible DPM gap junction sites (yellow).

We also visualized INX6 immunoreactivity in the mushroom body, where gap junctions essential for memory formation are formed between DPM and APL neurons (Wu et al., 2011). Using ExLLSM, we found that INX6 immunoreactivity was punctate in the mushroom body, with few if any clusters (Fig. 4G-H). These punctate signals colocalized with DPM and APL neurons (Fig. 4E-H). We found that DPM-associated INX6 and APL-associated INX6 contacted the APL and DPM membranes, respectively. However, we did not detect contact between DPMassociated INX6 and APL-associated INX6. It is unclear if the punctate INX6 we detected in these neurons labels functional gap junctions, hemichannels, or portions of heterotypic gap junctions. Previous findings indicated that the electrical coupling between DPM and APL neurons is mediated by heterotypic gap junctions composed of INX6 and INX7, respectively (Wu et al., 2011), which suggest that we may be visualizing part of the gap junctions formed between these neurons.

Although additional work is necessary to characterize the structure of hemichannels and homo- and heterotypic gap junctions in superresolution light microscopy, these data demonstrate the ability of our ExLLSM pipeline to detect electrical as well as chemical connections.

\section{Linking circuit structure to neurophysiology and behavior across individuals}

Individual variations in structural connectivity arise through ontogenetic, environmental, and stochastic processes. A major challenge in neuroscience is to understand how these structural variations relate to individual differences in circuit physiology, function, and behavior. There is not necessarily a one-to-one mapping between circuit connectivity, physiology, and output (Marder, 2011; Marder et al., 2015), and so a full investigation of the 
structure-function relationships in neural circuits requires that each be examined across many individuals. By enabling the rapid reconstruction of neural circuits at synaptic resolution, ExLLSM is ideally suited to this task. To evaluate this potential, we examined male courtship song in Drosophila, a highly stereotyped yet variable behavior that can be readily quantified and for which critical nodes in the underlying circuitry have been identified.
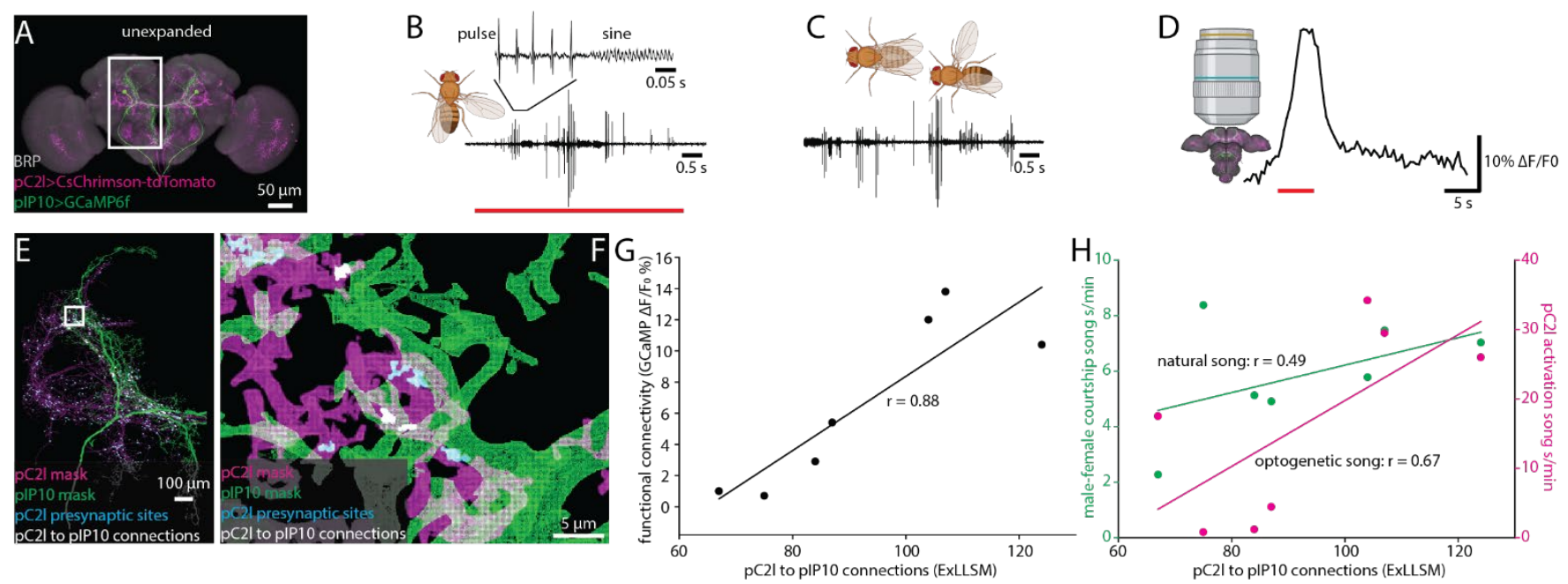

Figure 5 Correlating individual variability in structural connectivity, functional connectivity, and behavior. (A) Unexpanded brain of a male fly labeled to reveal all presynaptic sites (BRP, grey), GCaMP6f (green) in pIP10, CsChrimson (red) in pC2I. (B) Representative example of optogenetic activation of $\mathrm{pC2l}$ (red bar), resulting in the acute production of pulse and sine song. (C) Representative example of song produced by the male during a courtship bout with a female. (D) Representative example of the GCaMP6f response in pIP10 induced by optogenetic activation of pC2I. (E) Representative 8X view of a segmented pC2I, segmented pIP10, pC2I presynaptic sites, and the $p C 2 I$ to pIP10 connections as measured by pIP10 contact with pC2I presynaptic sites. (F) Zoom in on region in the rectangle from (E). $(G, H)$ ExLLSM connections plotted against (G) the strength of the functional connection between $p C 2 I$ and $p I P 10$, and $(H)$ song seconds/minute elicited by optogenetic $p C 2 I$ activation (magenta) and by a female fly (green). Linear regression and associated Pearson's Correlation Coefficient (r) plotted for each relationship.

We focused our analysis on two neuron types in the male brain that function in song production, pC2I and pIP10 (Fig. 5A). The male courtship song consists of a series of loud pulses, interspersed with bouts of continuous humming known as the sine song (Greenspan and Ferveur, 2000) (Fig. 5B-C). The pC2I neurons respond to both auditory and visual cues (Kohatsu and Yamamoto, 2015; Deutsch et al., 2019), and activating a subset of these neurons elicits pulse song acutely, followed by sine song post-activation (Deutsch et al., 2019). Here, using a different genetic driver line that labels $10 \mathrm{pC} 2 \mathrm{l}$ neurons in each hemisphere, we found that pC2I activation produced acute courtship song in most files with natural pulse and sine characteristics (Fig. 5 B, Sup. Fig. 4A-B). pIP10 cells are bilaterally-paired male-specific descending neurons. pIP10 activity is necessary and sufficient for the production of pulse song and, to a lesser extent, sine song, and influences song choice during courtship (von Philipsborn et al., 2011; Clemens et al., 2018; Calhoun et al., 2019; Ding et al., 2019).

To examine individual variability in the structure and function of the pC2I-pIP10 circuit, we expressed the red-light activated cation channel CsChrimson (Klapoetke et al., 2014) in pC2I neurons via a split-LexA line and the calcium indicator GCaMP6f (Chen et al., 2013) in pIP10 neurons via a split-GAL4 line (Ding et al., 2019) (Fig. 5A). In seven individual flies, we optogentically activated $\mathrm{pC2}$ and quantified the song generated (Fig. 5B), then paired a male with a female for 10 minutes and quantified the courtship song produced upon natural stimulation (Fig. 5C). Next, we removed the brain, optogentically activated pC2I and measured the calcium response in pIP10 (Fig. 5D), before 
finally using 8X ExLLSM to quantify any structural connectivity between pC2I and pIP10 (Fig. 5E-F). With this protocol, we could assess how courtship behavior, optogenetically-elicited behavior, functional connectivity, and structural connectivity are all related to each other across individual flies (Fig. 5G-H, Sup. Fig. 4 C-D).

We established that $\mathrm{pC2}$ cells are presynaptic to pIP10, counting an average of 92 connections. In these experiments, we had to rely on GCaMP6 as the pIP10 label rather than the membrane marker used in the previous experiments with SAG and $\mathrm{pC} 1$ cells, which likely further but consistently undercounts the number of connections. There was a strong linear relationship between the number of structural connections and the strength of the functional connection across animals (Pearson's $r=0.88, P=0.009$, Figure $5 G$ ). Structural connectivity between $p C 2$ I and pIP10 was less strongly correlated with optogenetically-elicited song (Pearson's $r=0.67, \mathrm{P}=0.09$, Fig. 5H), and least of all with naturally-stimulated song (Pearson's $r=0.49, \mathrm{P}=0.27$, Fig. 5H).

We also observed a strong linear correlation between the functional connection and optogenetic song (Pearson's $r=0.83, P=0.02$, Sup. Fig. 4D) more so than between structure and optogenetic song (Pearson's $r=0.67, P=0.09$, Fig. $5 G$ ) and functional connectivity and naturally-stimulated song (Pearson's $r=0.37, P=0.41$, Sup. Fig. 4D). These relationships are unlikely to merely reflect individual variations in expression levels of the GCaMP6 reporter, as there was no correlation between the baseline fluorescence of the GCaMP signal and the structural connection (Pearson's $r=-0.09, \mathrm{P}=0.85$, Sup. Fig. 4E).

As noted, courtship song consists of pulse and sine components, and previous evidence suggests that pC2I and pIP10 directly drive pulse song with indirect contributions to sine song production (Deutsch et al., 2019; Ding et al., 2019; Roemschied et al., 2021). This suggests that pC2I>pIP10 connectivity may have a stronger relationship to pulse than sine or total song. Indeed, separating song into its pulse and sine components revealed a stronger relationship between structure and optogenetic pulse song production (Pearson's $r=0.75, P=0.054$, Sup. Fig. 4C) compared to song production in the aggregate (Pearson's $r=0.67, P=0.09$, Fig. $5 \mathrm{G}$ ) or sine production alone (Pearson's $r=0.11, P=0.82$, Sup. Fig. $4 C$ ). These data are consistent with evidence that $\mathrm{pC} 2 \mathrm{I}$ and pIP10 activate sine song less directly (Roemschied et al., 2021), and demonstrates that ExLLSM allows an individually variable feature of behavior to be correlated with individual variations in structural connectivity.

\section{Discussion}

Our goal in this project was to develop an ExLLSM pipeline for the rapid and sparse reconstruction of neural circuits. We used Drosophila due to the availability in this species of reference connectomes (Takemura et al., 2015, 2017; Bates et al., 2020; Scheffer et al., 2020) and genetic tools for highlighting cells and molecules of interest (Jenett et al., 2012; Andlauer et al., 2014; Chen et al., 2014b; Tirian and Dickson, 2017; Dionne et al., 2018). The protocols we have developed allow a single sample to be prepared and analyzed within a week and can be readily multiplexed to process several samples in parallel. Although LLSM provides an excellent combination of imaging resolution and speed with limited photobleaching (Gao et al., 2019), other light-sheet microscopes can also be utilized to image large volumes of $8 \mathrm{X}$ expanded samples. As such, our method relies on relatively affordable microscopy and computational resources that are widely available, bringing connectomics research within reach of smaller labs. In establishing this pipeline, we developed new software tools to visualize and analyze the high-resolution multi-terabyte datasets generated. These tools are freely available (Lillvis, 2021; Lillvis et al., 2021; Rokicki and Kawase, 2021; Rokicki and Lillvis, 2021) and have been designed with efficiency and flexibility in mind. In particular, provided a suitable ground truth data set is available, the convolutional neural network models for synapse and neuron segmentation can be retrained and the entire computational pipeline applied to samples obtained from any species or microscope. 
Many further developments will be spurred, we anticipate, by the power and low entry cost of ExLLSM-based circuit analysis. With the development of a membrane label that leaves fewer gaps in the expanded sample, automated single neuron segmentation should become feasible (Januszewski et al., 2018) and would allow connectivity to be assessed at the level of single cells rather than single cell types, as we have done here. Such methods would likely also allow more densely labelled neurons to be traced, ultimately perhaps even entire connectomes (Gao et al., 2019). A further improvement would come through the generation of reagents to visualize specific components of chemical and electrical synapses, ideally in the form of genetic tools that label endogenous molecules in a cell-type-restricted manner (Chen et al., 2014b). Because ExLLSM enables correlative structural and functional studies, probes that reveal the functional state as well as the location of such molecules would be particularly valuable.

We have shown here that comparative ExLLSM can be used to reveal state-dependent differences in neuronal connectivity. More generally, the method is ideally suited to explore how genetic, environmental, and stochastic processes work together to create individual differences in neuronal connectivity. In combination with new methods to genetically identify and label homologous neurons across species (Stern et al., 2017; Tanaka et al., 2017; Seeholzer et al., 2018; Ding et al., 2019; Auer et al., 2020), this approach will also enable new studies on circuit and behavior evolution. By facilitating the collection of both functional and structural data from the same samples, our ExLLSM pipeline also allows individual and species differences to be correlated with differences in neurophysiology and behavior. Our ExLLSM approach to neural circuit reconstruction thereby fills a critical methodological gap in exploring the links between genes, physiology, and behavior, and should be a powerful tool in efforts to understand how connectomes work and how they evolve.

\section{Acknowledgments}

We thank Ramya Kappagantula and Claire Managan for help with generating presynaptic site ground truth data; Damien Alcor and Michael DeSantis for help with imaging; Eric Betzig for input on the LLSM build; Igor Negrashov for mechanical design of the LLSM; Yoshi Aso, Stephan Sigrist, Aljoscha Nern, Yichun Shuai, Glenn Turner, and Gerry Rubin for transgenic fly reagents; the Janelia FlyLight project team for generating the images of unexpanded fly brains; David Ackerman for helpful data analysis discussions and help with image stitching at Janelia; Monique Copeland for help with ExM sample preparation; Gudrun Ihrke for helpful immunohistochemistry discussions; Meng-Hsuan Chiang and Chia-Lin Wu for the INX6 antibody; Ken Carlile, Rob Lines, Habib Bukhari, and Stephan Preibisch for help with data analysis on the Janelia LSF cluster; Sean Murphy for data analysis discussions; Lou Scheffer for $\mathrm{L} 2$ data from the 7-column optic lobe FIB-SEM dataset; Stuart Berg and the FlyEM project team for SAG, pC1, and DA1-IPN data from the hemibrain FIB-SEM dataset; Philipp Schlegel and Greg Jefferis for DA1-IPN data from the TEM dataset; Fei Wang for helpful discussions; Henry Ngo for workstation help; David Stern for helpful comments on the manuscript; and Ben Arthur, Emily Behrman, and Elizabeth Kim for help with courtship song recording and analysis.

\section{Author Contributions}

J.L.L. and B.J.D. supervised the project and wrote the manuscript with input from coauthors. P.W.T. created and supervised use of the 8X ExM protocol. J.L.L. and A.H. prepared the ExM samples. J.L.L. imaged, processed, and analyzed all ExLLSM data. J.C. provided essential discussions on ExLLSM imaging, processing, and analysis and built the LLS microscope with input from D.E.M. K.W. provided essential genetic reagents. C.G. and K.R. adapted ExLLSM stitching and analysis code for distribution and generated Nextflow workflows. T.K. developed VVD Viewer for ExLLSM data and wrote VVD file conversion code. H.O. developed VVD Viewer for ExLLSM data, wrote VVD file conversion code, and wrote image processing tools including MIP creation, ROI cropping, 3D component 
connecting, and intensity thresholding. X.D. wrote the automatic synapse classification and post-classification segmentation, assignment, and analysis code. L.M. wrote the automatic neuron segmentation code. I.P. and S.S. wrote the flat-field, stitching, and N5 visualization code. I.P. wrote the N5 file conversion code and the N5 connected components analysis tools including pixel shape change, intensity thresholding, and component size thresholding. R.G. and J.B. wrote the deconvolution code. R.G. provided helpful discussions on ExLLSM imaging and processing and provided initial $4 \mathrm{X}$ ExM protocols and samples with E.S.B. D.E.M. created the instrument control software. J.L.L. conducted and analyzed all behavior experiments and calcium imaging experiments. J.L.L. produced all figures and movies.

\section{Materials and Methods}

\section{Experimental animals}

Unless noted otherwise, flies were raised on standard cornmeal-agar based medium on a 12:12 light/dark cycle at $25^{\circ} \mathrm{C}$. Detailed information on fly genotypes, sex, housing, and age for each experiment are indicated in the relevant section below and in Table 1.

\section{Genetic reagents}

LexA, split-GAL4, and split-LexA lines used in this study have constructs inserted at the attP40 or attP2 landing sites (Tirian and Dickson, 2017; Dionne et al., 2018), unless noted otherwise (Table 1). Unpublished LexA, p65ADZp, ZpGAL4DBD, and ZpLexADBD lines labelling neurons of interest were identified using a color depth maximum intensity projection mask search (Otsuna et al., 2018). The expression of driver lines was examined with a UAS or LexAop reporter by immunofluorescence staining and confocal microscopy (https://www.janelia.org/project-team/flylight/protocols). For split lines, the combinations of p65ADZp and ZpGAL4DBD or p65ADZp and ZpLexADBD that gave the most specific expression patterns were stabilized by putting the two hemi-drivers in the same flies, and SS and SL (denoting stable split-GAL4 or split-LexA, respectively) numbers were assigned. SS and SL combinations were checked for expression in the same fly to ensure that no off-target neuron expression was found in the overlapping regions of interest due to unintended interactions between SS and SL p65ADZp, ZpGAL4DBD, ZpLexADBD components.

\section{Immunohistochemistry}

For ExLLSM experiments, brains were dissected in external saline composed of (in mM) $103 \mathrm{NaCl}, 3 \mathrm{KCl}, 5 \mathrm{~N}-$ tris(hydroxymethyl) methyl-2-aminoethane-sulfonic acid, 10 trehalose dihydrate, 10 glucose, 26 NaHCO3, 1 $\mathrm{NaH} 2 \mathrm{PO} 4,4 \mathrm{MgCl}$, $3 \mathrm{KCl}, 2$ sucrose, and $1.5 \mathrm{CaCl} 2$ (280-290 mOsm, pH 7.3; all components from Sigma). All subsequent washes and incubations were conducted on a rocker or rotator. After dissection, samples were fixed in $2 \%$ formaldehyde (Electron Microscopy Solutions, $20 \%$ stock diluted in external saline) at room temperature for 55 minutes, washed in three 20 minute PBST (0.5\% Triton X-100 in $1 \mathrm{X}$ phosphate buffered saline) washes, and blocked using $5 \%$ normal goat serum (diluted in PBST) for 90 minutes. After blocking, samples were incubated in primary antibodies at $4^{\circ} \mathrm{C}$ for 2-3 days, washed for 2-5 total hours at room temperature in at least five PBST washes, and then incubated in secondary antibodies at $4^{\circ} \mathrm{C}$ for 2 days. All antibodies and concentrations are listed in Table 1. Finally, samples were washed for 2-5 total hours at room temperature in at least five PBST washes and stored in $1 \mathrm{X}$ phosphate buffered saline at $4^{\circ} \mathrm{C}$ until they were prepared for expansion, which occurred within 24 hours. Unexpanded images shown were prepared by the Janelia Fly Light Project Team. Unexpanded samples were prepared in a largely similar manner to expanded samples and mounted in DPX on a glass slide. For detailed 
unexpanded brain dissection, immunohistochemistry, and DPX mounting protocols, see https://www.janelia.org/project-team/flylight/protocols.

\section{X expansion}

Acryloyl-X, SE (6-((acryloyl)amino)hexanoic acid, succinimidyl ester; here abbreviated AcX; Thermo-Fisher) was resuspended in anhydrous DMSO at a concentration of $10 \mathrm{mg} / \mathrm{mL}$, aliquoted, and stored frozen in a desiccated environment. AcX stock solution was diluted in $1 x P B S$ to a final concentration of $0.1 \mathrm{mg} / \mathrm{mL}$ AcX. Specimens were incubated in this $0.1 \mathrm{mg} / \mathrm{mL} A c X$ solution overnight at room temperature. Gelation chambers were created by adhering silicone gaskets (e.g. Sigma, GBL665504) to poly-lysine-treated glass slides. Specimens were immobilized on the poly-lysine-treated surface, at least $2 \mathrm{~mm}$ away from the silicone surface.

A $4 \mathrm{M}$ sodium acrylate stock solution was prepared by combining $5.5 \mathrm{~mL}$ acrylic acid (Sigma, 147230), $4.5 \mathrm{~mL}$ water, and $7.2 \mathrm{~mL} 10 \mathrm{M} \mathrm{NaOH}$ in a fume hood, then adding $1 \mathrm{M} \mathrm{NaOH}$ until the $\mathrm{pH}$ reached 7.75-8, and finally adding water up to $20 \mathrm{~mL}$. This solution should be clear. Monomer solution (1xPBS, $1 \mathrm{M} \mathrm{NaCl}, 1.84 \mathrm{M}$ sodium acrylate, $2.5 \%$ acrylamide, $0.05 \% \mathrm{~N}, \mathrm{~N}^{\prime}$-methylenebisacrylamide) was mixed, frozen in aliquots, thawed fully, vortexed, and cooled on ice before use. Concentrated stocks of the initiator ammonium persulfate (APS, $10 \mathrm{wt} \%$ ), accelerator tetramethylethylenediamine (TEMED, 10 vol\%), and inhibitor 4-hydroxy-2,2,6,6tetramethylpiperidin-1-oxyl (4-HT, $0.5 \mathrm{wt} \%$ ) were prepared as concentrated stock solutions, which were frozen in aliquots and then fully thawed and vortexed before use. Initiator, accelerator, and inhibitor stock solutions were added to the monomer solution at a ratio of $2 \mathrm{uL}$ each per $94 \mu \mathrm{L}$ monomer solution to produce gelation solution. AcX-anchored specimens were washed $3 \times 10$ min in gelation solution, on ice. Gelation chambers were sealed with a $22 \mathrm{~mm}$-square coverslip, excess gelation solution was removed, and the sealed chambers were transferred to $37^{\circ} \mathrm{C}$ for 2 hours, protected from light, for gelation.

Proteinase K (New England Biolabs) was diluted 1:100 to 8 units/mL in digestion buffer (50 mM Tris (pH 8), $1 \mathrm{mM}$ EDTA, $0.5 \%$ Triton $\mathrm{X}-100,500 \mathrm{mM} \mathrm{NaCl}$ ) to produce proteinase solution. Gels were recovered from chambers, trimmed close to the specimens, and incubated fully immersed in proteinase solution overnight at room temperature, with shaking. Gels were washed in 1xPBS for $30 \mathrm{~min}$ to remove proteinase K. Digested gels were next incubated on ice, with shaking, in at least a 10 -fold excess volume of gelation solution $2 \times 10$ min, with APS omitted in the first two incubations. A $5 \mathrm{~mL}$ syringe filled with silicone grease was used to apply four dabs of grease per specimen to glass slides, at the corners of one $\sim 20 \mathrm{~mm}$ square per specimen. Each gel was transferred to the middle of one of these squares and covered with a $22 \mathrm{~mm}$-square coverslip. This coverslip was pressed down to gently but fully contact the gel, while being held in place by the dabs of vacuum grease. Excess gelation solution was backfilled into the resulting chamber to impede access of atmospheric oxygen to the gel. The completed chamber was moved to the $37^{\circ} \mathrm{C}$ incubator for 2 hours for gelation. The resulting doubly-gelled specimen was recovered from the chamber, and excess gel was trimmed away, followed by staining in $0.2 \mathrm{mg} / \mathrm{mL}$ DAPI in $1 \times$ PBS for $2 \mathrm{hr}$. The gel was incubated in $\sim 50 \mathrm{~mL}$ of doubly deionized water for 12-24 hours to expand.

\section{Lattice light-sheet imaging}

Sample mounting was highly similar to Gao R et al., 2019 with some slight modifications. A $12 \mathrm{~mm}$ round glass coverslip was brushed with a solution composed of poly-L-lysine hydrobromide $(0.078 \%$ weight/volume; Sigma Aldrich: P1524) and Kodak Photo-Flo 200 (0.2\% volume/volume; Electron Microscopy Sciences: 74257) and allowed to dry. Using a razor blade, $8 \mathrm{X}$ expanded gels were trimmed close to the tissue sample in $\mathrm{x}, \mathrm{y}$, and $\mathrm{z}$ by viewing DAPI staining under a wide field microscope (Olympus MVX10). Liquid around the gel was wicked away with a Kimwipe (Kimtech Science) and the gel was transferred onto the dry, coated coverslip attached to a sample 
holder by metal clamps. The sample holder was attached to the LLSM sample chamber which was filled with 1 $\mathrm{mM}$ Tris base, which was used to reduce sample shrinking (possibly due to acidification from atmospheric $\mathrm{CO}_{2}$ ) during long imaging runs.

The imaging region of interest (ROI) was identified by taking a snapshot of the neuron fluorescence using a wide field camera mounted under the sample bath. An ROI was drawn and the $x, y$ coordinates of the ROI relative to the stage position were calculated. The $z$ coordinates were determined by scanning through the sample and identifying the upper and lower bounds of the region of interest. The z-offset for each wavelength, which sets the precise position of the light sheet relative to the detection plane of the objective, was determined by taking small z-stacks of each channel, re-slicing the stack, and creating a MIP of the re-sliced stack to view the symmetry of the XZ PSF. Offset was adjusted until the PSF was symmetric, similar to the procedure described in Figure 5 of Gao R et al., 2019. Adjustments of up to $1 \mu \mathrm{m}$ are common to account for the slight mismatch between the refractive index of the gel and buffer.

LLSM hardware and software setup and control were near identical to the "LLSM optimized for expanded samples" described in Gao R et al., 2019, with some slight modifications described here. Samples were excited by 488, 560, and $642 \mathrm{~nm}$ lasers. Emission was split between two detection cameras using a 561nm dichroic mirror (Semrock: Di03-R561-t1-25×36). In front of the green camera, FF02-525/40-25, FF01-432/515/595/730-25, and NF03405/488/561/635E-25 emission filters (Semrock) were used. In front of the red camera, FF01-440/521/607/70025 and NF03-405/488/561/635E-25 emission filters (Semrock) were used. All samples were imaged in objective

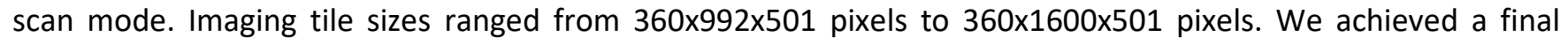
resolution of $30 \times 30 \times 100 \mathrm{~nm}$, as calculated from the FWHM of the $560 \mathrm{~nm}$ PSF in XYZ. This is slightly lower than the best possible resolution for the beam NA (0.517-0.55); the light sheet was tuned to be slightly thicker to allow for sample variation and small amounts of instrument drift.

For large samples with non-rectangular processes, imaging tiles with no signals were automatically avoided by the software using the "intelligent tiling" technique described in Gao R et al., 2019. This strategy significantly reduced imaging time compared to standard tiling while capturing the signal of interest. The sample bath remained static throughout imaging, but imaging was occasionally paused to refill the bath to ensure the sample remained submerged during long imaging runs.

\section{Image processing and analysis overview}

The goal of image analysis here was to quantify synaptic connectivity between neuron types (e.g. neuron 1 and neuron 2) labeled by transgenic fly lines. To accomplish this, image tiles were processed and stitched. Then fluorescently labeled neurons, presynaptic, and in some cases, postsynaptic sites were segmented. Next, a colocalization analysis assigned the classified pre and/or postsynaptic sites to segmented neuron masks. Finally, an additional colocalization analysis identified connections between the neurons by finding presynaptic sites from neuron 1 that contact neuron 2 or postsynaptic sites from neuron 2 that contact neuron 1 presynaptic sites (Sup. Fig. 2).

To maximize accessibility and portability of our processing and analysis tools, we are distributing executable Docker containers (Merkel, 2014) for all of the code, making it easy to run the code across a wide range of systems through the use of Singularity (Kurtzer et al., 2017). The use of Nextflow further reinforces that goal by allowing our computational workflows to execute on any compute cluster or cloud, including but not limited to IBM 
Platform LSF, SLURM, and AWS Batch. By assembling the workflows into Nextflow pipelines, we also minimize the dependencies that are necessary for the user to install and provide a consistent command-line interface for invoking workflows and specifying runtime options. Each step of the pipeline is described below. Code and additional documentation to run all steps of the analyses described can be found at https://github.com/JaneliaSciComp/exllsm-circuit-reconstruction (Lillvis, 2021; Lillvis et al., 2021; Rokicki and Kawase, 2021; Rokicki and Lillvis, 2021).

\section{Image preprocessing and stitching}

Image preprocessing (flat-field correction and deconvolution), stitching, and stitched N5 (https://github.com/saalfeldlab/n5) and TIFF series exports were conducted as in Gao R et al., 2019. Default preprocessing and stitching parameters were also identical to those listed in Gao et al., 2019. We include all of these published preprocessing and stitching steps (https://github.com/saalfeldlab/stitching-spark) in our analysis pipeline (https://github.com/JaneliaSciComp/exllsm-circuit-reconstruction). The entire preprocessing and stitching pipeline can be run as a workflow with flexible parameter setting options, or each step can be run independently.

\section{Visualization}

For 3D visualization, preliminary image analysis, user guided semi-automatic neuron segmentation, ground truth data generation, and video creation we extended the free and open source VVD Viewer software (https://github.com/JaneliaSciComp/VVDViewer) (Wan et al., 2012) to handle high-resolution big image (data sizes $\leq 5 \mathrm{~TB} /$ channel tested) light microscopy data sets like those generated by ExLLSM. N5 directories generated by the stitching pipeline can be opened directly in VVD Viewer. For image analysis and segmentation, we recommend using multi-scale pyramid VVD Viewer files which are more efficiently transferred across the network and faster to load into GPU memory. We include workflows to convert N5 or TIFF series to VVD Viewer pyramid files that can be run locally or on a compute cluster. A detailed manual for running VVD Viewer, creating VVD Viewer files, and analyzing ExLLSM data in VVD Viewer can be found at https://github.com/JaneliaSciComp/exllsm-circuit-reconstruction.

Small image crops were used to generate ground truth data for training the synapse classifier (see Automatic synapse detection). The Fiji plugin " $\mathrm{n} 5$-ij" was utilized to open stitched N5 directories and make these crops (https://github.com/saalfeldlab/n5-ii).

\section{Semi-automatic neuron segmentation}

The first task to accomplish in order to quantify synaptic connectivity between neurons was to segment the neurons of interest in the fluorescently labeled neuron images. The primary barrier to this was the large size of the image data. Manually segmenting neurons in the multi-terabyte high-resolution images is a very slow process and existing methods and software to segment 3D light microscopy images did not transfer well to the large ExLLSM data sets. Using clean genetic fly line reagents - where no off-target neurons are labeled in the region of interest - may allow for basic image processing strategies (i.e. thresholding and size filtering) to be utilized for at least preliminary segmentation. However, this was not sufficient in most cases, and more sophisticated image segmentation strategies needed to be employed.

Therefore, we extended the VVD Viewer software (https://github.com/JaneliaSciComp/VVDViewer) (Wan et al., 2012) to semi-automatically segment multi-terabyte ExLLSM data sets. See https://github.com/JaneliaSciComp/exllsm-circuit-reconstruction for a detailed manual on segmenting neurons in 
ExLLSM datasets using VVD Viewer. Briefly, we used the VVD Viewer Component Analyzer tool to automatically and rapidly (seconds) segment down sampled ExLLSM neuron signals from background signal based on a combination of voxel intensity and connected component size thresholds. This tool individually segments disconnected components (neurons, synapse, etc.), but does not separate components if they are connected or touching. Therefore, this tool works well to automatically segment images that label individual neurons, multiple disconnected neurons, or multiple connected neurons that are being analyzed as a unit (e.g. a class of neurons; e.g. DA1-IPN in Fig. 1B-D and 2K-P). However, to segment individual neurons that contact each other, the Component Analyzer tool can be used for initial segmentation from background, but the user will need to manually segment individual neurons (e.g. Fig. 1D, 2B, E-J).

The segmentation result generated by Component Analyzer was manually edited in VVD Viewer until the final mask appropriately segmented the neuron(s) of interest. This final segmented mask was saved as a TIFF series that retains the original voxel size and intensity values at 8 bit (Sup. Fig. 1).

In order to allow fast segmentation from background and 3D editing of the large ExLLSM datasets, Component Analyzer runs on a downsampled VVD pyramid. Consequently, the segmented neuron TIFF series will generally over mask the neuron on its edges (Sup. Fig 1C, F, I, L). We found that the best way to correct this was to apply a pixel intensity threshold the TIFF series. Thresholding levels were determined by generating a MIP of the segmented mask TIFF series. In most cases, the thresholding value generated by the Huang or Li method removed the edge over masking and generated a mask that was true to the neuron signal (Sup. Fig. 1C, F, I, L). However, in some cases, these values were too low and a manually determined pixel intensity threshold value that accurately masked the neurons was used.

At this stage, we have generated a mask that is true to the fluorescent signal of the neuron. However, at $8 \mathrm{X}$, the fluorescent signal along neurons is not completely continuous due largely to gaps in fluorophore or tag expression and/or antibody labeling along the neuron (Gao et al., 2019) (Sup. Fig. 1D, G, J, M). These gaps in signal were filled using a flexible, 3D component connecting algorithm. We connected gaps of 20 voxels or less, and iterated this process four times. This process reliably connected disconnected neuron components that were clearly part of a continuous neuron with minimal unwanted connections (Sup. Fig. 1D, G, J, M).

Finally, the components of this mask were analyzed, pixel shape was converted from diamond to box (to further connect any directly neighboring pixels), and disconnected components smaller than 2000 voxels were removed (Sup. Fig. 1D, G, J, M). The result of these steps creates a binary mask of the neuron signal in the imaging volume. We include all of the post-VVD Viewer mask processing steps (MIP creation, voxel intensity thresholding, 3D gap filling, connected component analysis, voxel shape changing, and size filtering) in our ExLLSM analysis pipeline tools. Each of these steps can be run independently, and therefore utilized for other image processing needs, or as a single workflow with flexible parameter inputs. See https://github.com/JaneliaSciComp/exllsm-circuitreconstruction for more details, usage examples, and tutorials.

\section{Automatic neuron segmentation}

Although the semi-automatic segmentation method described above is relatively fast and can be done with little manual intervention, we sought to determine whether we could accomplish this task automatically. To do this, we generated a 3D U-Net convolutional neural network (Çiçek et al., 2016) to automatically segment neural signals from non-specific antibody labeling and noise. For the purposes of this manuscript, we are focusing on segmenting all neurons labeled as a group as opposed to segmenting individual neurons from each other. In order to secondarily segment individual neurons from each other, VVD Viewer can be utilized to do so manually. 
Ground truth data was generated via the semi-automatic neuron segmentation process described above. Two $1024 \times 1024 \times 512$ pixel crops were made from 17 samples (representing 7 different neuron classes) for training. We used these data crops to train the U-Net for 150 epochs until the loss, accuracy, and error rates plateaued.

We evaluated this network by running it on full image volumes in five brain samples that were not included in the training. The output of the U-Net is a probability array with pixel values between 0 and 1 . The entire automatic neuron segmentation pipeline included post-U-Net pixel intensity thresholding (here, a 0.8 threshold was used) and size filtering to remove components smaller than 2000 pixels. We compared the results of the entire automatic neuron segmentation pipeline to semi-automatically segmented ground truth data of these data sets. Because most of the pixels contain no neural signals, $1024 \times 1024 \times 500$ pixel crops were made in regions where ground truth data was present. On these crops, the average precision was $95 \%$ and average recall was $79 \%$ (Sup. Fig. 3).

Notably, off-target neurons present in samples were included in the results of the automatic neuron segmentation pipeline. In some instances, this would not affect connectivity analyses because there is no overlap between these off-target neurons and the pre- or postsynaptic neurons of interest. In many other instances these off-target neurons would need to be removed before further analyses. Therefore, we elected to use the semi-automatic neuron segmentation via VVD Viewer strategy for all data analysis here.

However, this automatic approach was fast (2TB 15000x8000x10000 pixel image volumes were segmented in just 10 minutes on the Janelia compute cluster) and worked relatively well despite limited training. Therefore, developing this approach for future work is likely to improve analysis efficiency. We include all code for training and evaluating the neuron segmentation U-Net and the trained model used here (https://github.com/JaneliaSciComp/exllsm-neuron-segmentation) (Lillvis, 2021). Additional details, instructions, and workflows for running automatic neuron segmentation can be found at https://github.com/JaneliaSciComp/exllsm-circuit-reconstruction.

\section{ROI cropping}

Even when using intelligent tiling to reduce the acquisition of image tiles without neuron signals, it was often the case that a significant image volume was present outside of the neurons of interest. Therefore, after segmenting the key neuron(s), a 3D region of interest (ROI) was identified by stepping through VVD mask TIFF series in $\mathrm{z}$ in Fiji (Schindelin et al., 2012). The neuron masks and stitched TIFF series were then cropped based on a 2D ROI generated in Fiji, and the first and last slice of the 3D ROI. All subsequent steps (VVD mask post-processing and connectivity analyses) were conducted on the sub volumes generated after cropping. Analyzing these sub volumes significantly reduced computing time and expense. We include code to accelerate this process that can be run locally or submitted to a compute cluster (https://github.com/JaneliaSciComp/exllsm-circuit-reconstruction).

\section{Automatic synapse classification}

Presynaptic sites can be identified as clusters of BRP proteins (Ehmann et al., 2017). Using 8X ExLLSM and labeling BRP with the nc82 antibody (Wagh et al., 2006) or the STaR-BRP reporter (Chen et al., 2014b), discrete clusters of fluorescent antibodies were present that, as expected (Schneider-Mizell et al., 2016), varied significantly in shape and size across the Drosophila brain (Figure 1H-K). We tested using ilastik (Sommer et al., 2011), a 3D VGG shaped neural network (Simonyan and Zisserman, 2014), and 3D U-Net shaped neural network (Çiçek et al., 2016) to segment these heterogeneous structures from non-specific antibody labels and background signals. On our data, we found that the neural networks performed better than ilastik and similarly to each other, and that the U-Net 
was faster than the VGG. Therefore, we elected to train a U-Net convolutional neural network to automatically classify presynaptic sites.

To generate ground truth data for training the U-Net, we made $100 \times 100 \times 100$ and $500 \times 500 \times 500$ pixel crops of BRP staining (as labeled using the nc82 antibody) using the Fiji N5 Viewer. We considered clusters of three or more BRP labels in close proximity that fell along a common plane to be presynaptic sites. We semi-automatically segmented these presynaptic sites from non-specific antibody labels and background signals using VVD Viewer. This semi-automatic segmentation was accomplished similarly to semi-automatic neuron segmentation: the VVD Viewer Component Analyzer tool was used to extract signal from background followed by manual inspection of each potential presynaptic site. In total, we segmented over 10,000 presynaptic sites in image crops from 25 different brains. Crops were made from the optic lobe, mushroom body, lateral horn, central complex, antennal lobe, and protocerebrum.

We used these raw image data crops and manually segmented presynaptic sites to train the U-Net for 3000 epochs until the loss, accuracy, and error rates plateaued. The entire synapse classification and assignment pipeline includes a post-U-Net processing workflow. This post-U-Net workflow includes a watershed segmentation step to segment individual synaptic sites and a size filter to remove connected components below a given size threshold. For presynaptic sites labeled by nc82 or STaR-BRP, objects smaller than 400 pixels were removed.

We evaluated the results of this synapse detection pipeline (including post-U-Net watershed segmentation and 400 pixel size thresholding) by running it on data crops of BRP labeled by nc 82 from the optic lobe, protocerebrum, and lateral horn of three brain samples that were not included in the training. We compared these results to the manually segmented ground truth data (2300 presynaptic sites) of these image volumes. The final synapse detection pipeline had an average precision of $94 \%$ and recall of $88 \%$ (Fig. 1L-Q).

In addition to labeling presynaptic sites by visualizing BRP, we labeled putative cholinergic postsynaptic sites by visualizing Drep2 (Andlauer et al., 2014) in pC1 neurons using 10XUAS-smFP-HA-drep2-sv40. This labeling strategy reports overexpressed, not endogenous, levels of Drep2 protein that appear as punctate signals or small clusters of punctate signals, that were grossly similar to presynaptic sites structures (Fig. 3K, M-O). Due to the similarity of the labels we were seeking to analyze, we tested whether the U-Net model trained on presynaptic site data could be used to classify postsynaptic receptors as labeled by 10XUAS-smFP-HA-drep2-sv40. We also utilized the postU-Net processing steps with a 200 pixel minimum size filter on these postsynaptic sites. Upon visual inspection, the classifier worked well. Clusters of Drep2 were grouped together or separated similarly to BRP as anticipated. Therefore, we used the same classifier to identify presynaptic sites and postsynaptic sites labeled by Drep2.

Finally, we used the same strategy to classify putative gap junctions or hemichannels as labeled by innexin 6 . Here, the INX6 label appeared in two forms: as punctate signals or as larger clusters or plaques. We used the same UNet model trained on presynaptic sites with post-U-Net watershed segmentation and a size filter of 200 pixels. This strategy worked well to, preliminarily at least, associate INX6 with segmented neuron masks and to classify potential gap junctions.

In principle, this detector should work well on detecting fluorescent punctate signals and clusters labeling other presynaptic, receptor, or gap junction proteins. However, the detector can be readily trained to classify any specific label if sufficient ground truth data can be generated.

We include the trained model used here for classifying synaptic sites, code and instructions to train the classifier, and code and instructions to calculate performance of the classifier (https://github.com/JaneliaSciComp/exllsm- 
synapse-detector) (Rokicki and Lillvis, 2021). These components can be run locally or on a compute cluster, and can be run independently or as part of several common use workflows described below (https://github.com/JaneliaSciComp/exllsm-circuit-reconstruction).

\section{Synapse connectivity analysis workflow}

With segmented neuron masks and a trained model to classify synaptic sites in hand, the connectivity analysis pipeline can be used. To analyze large ExLLSM imaging volumes, we partitioned the images into 512x512x512 pixel sub-volumes, analyzed these sub-volumes in parallel, and then re-stitched the analyzed results.

We developed three common use analysis workflows that support flexible parameter inputs (Sup. Fig. 2). Workflow A quantifies presynaptic sites in neuron 1 and connections from neuron 1 to neuron 2 based on neuron 1 presynaptic site contact with the neuron 2 membrane if presynaptic sites are labeled ubiquitously (as with BRP labeled via the nc82 antibody) (e.g. Fig. 3I-O, Fig. 5E-F). Workflow B quantifies presynaptic sites in neuron 1 and connections from neuron 1 to neuron 2 based on neuron 2 postsynaptic site contact with neuron 1 presynaptic sites if presynaptic sites are labeled ubiquitously and postsynaptic sites are labeled specifically in neuron 2 (as with 10XUAS-smFP-HA-drep2-sv40 labeling) (e.g. Fig. 3B-F). Workflow B can also be used if postsynaptic sites are labeled ubiquitously (e.g. with an antibody against a receptor protein) and presynaptic sites are labeled specifically in a neuron (as with STaR-BRP). Workflow C quantifies all pre- or postsynaptic sites in a given volume and can quantify synaptic sites in a single neuron mask (e.g. Fig. 2).

These workflows are composed of 2 to 4 stages. At stage 1 of Workflows A-C, pre- and/or postsynaptic label data is classified by the trained U-Net model (Sup. Fig. 2A-C). At stage 2 of Workflow C, classified synaptic sites are segmented and size filtered. At stage 2 of Workflows $A-B$, the neuron mask and synaptic site subvolumes are compared; any subvolumes without a neuron mask present are ignored. Subvolumes where the neuron mask is present are analyzed further. Synaptic sites are run through watershed segmentation, size filtered, and assigned to a neuron mask via colocalization (Sup. Fig. 2A-B). This colocalization value is flexible - the centroid of the synapse or any percentage of the synapse overlap with the neuron mask can be used. We found that synaptic sites were correctly assigned to neurons if $50 \%$ or more of the synaptic site volume overlapped with the neuron mask. Therefore, we used a value of $50 \%$ for all data here. At stage 3 of Workflows $A-B$, connections were quantified via either neuron 1 presynaptic site contact with neuron 2 (Workflow $A$ ) or neuron 2 postsynaptic site contact with neuron 1 presynaptic sites (Workflow B) (Sup. Fig. 2A-B). Here, we used a colocalization of $0.1 \%$-essentially any contact between pre- and postsynaptic sites was considered a connection. Finally, at stage 4 for Workflow $B$, the neuron 1 presynaptic sites that contact $(0.1 \%$ colocalization) neuron 2 postsynaptic sites are identified and quantified (Sup. Fig. 2B). The synaptic sites and connections identified each stage are collated into a stage-specific csv file that includes the shape and size of the synaptic site and the position of the site or connection. Additionally, images of the results from each stage are exported in N5 format with options to automatically generate TIFF series and VVD Viewer pyramid files. Examples of how to flexibly utilize each of these workflows or to run steps of each workflow independently are detailed at GitHub (https://github.com/JaneliaSciComp/exllsm-circuit-reconstruction).

\section{Behavior experiments}

For experiments comparing SAG>pC1 connectivity in virgin and mated females (Fig. 3H), virgin female flies were collected shortly after eclosion. After 2-3 days, individual females were either transferred to a new vial without male flies or to a new vial with male flies. After 2 additional days, virgin and mated females were dissected, 
processed for immunohistochemistry, expanded, imaged, and analyzed to quantify structural connectivity. Virgin and mated females were confirmed as such by the lack or presence of moving larvae in the final vial, respectively.

For pC2l>pIP10 structure-function experiments (Fig. 5, Sup. Fig. 4), naïve males were collected shortly after eclosion and single-housed for 4-6 days before beginning behavior experiments. Crosses and male aging were conducted in the dark on standard media containing $0.4 \mathrm{mM}$ trans-retinal.

Song behavior experiments were conducted in a song recording apparatus described previously (Arthur et al., 2013; Ding et al., 2019). pC2I neurons were optogentically activated in isolated males. For CsChrimson activation, constant $635 \mathrm{~nm}$ light was applied. Pulse width modulation with a $100 \mathrm{kHz}$ frequency was used to adjust light intensity. A stimulation cycle consisted of $25 \mathrm{~s}$ OFF and $5 \mathrm{~s}$ ON at the following light intensities: $2.5 \mu \mathrm{W} / \mathrm{mm}^{2}, 5.3$ $\mu \mathrm{W} / \mathrm{mm}^{2}, 8.0 \mu \mathrm{W} / \mathrm{mm}^{2}, 10.8 \mu \mathrm{W} / \mathrm{mm}^{2}$, and $15.6 \mu \mathrm{W} / \mathrm{mm}^{2}$. This cycle was repeated three times and response were averaged across trials. Pulse and sine song events were annotated manually. Because the only light intensity to reliably elicit song behavior was the maximum $15.6 \mu \mathrm{W} / \mathrm{mm}^{2}$, behavior from this light intensity was used for comparisons in Figure 5 and Supplemental Figure 4.

After optogenetic activation experiments, a 1-2 day old virgin female was paired with each male and audio was recorded for 10 minutes in dim blue light. The song generated in these assays was analyzed using SongExplorer (Arthur et al., 2021). A convolutional neural network trained for 3,024,000 steps on over 5000 pulse, 3000 sine, 2000 inter-pulse interval, and 2000 other manually annotated events was used to classify song. This trained model exhibited $\sim 80 \%$ precision and recall on novel song data from flies not included in the training data set.

Each fly was catalogued and the functional synaptic connection from pC2I and pIP10 was tested the following day via calcium imaging.

\section{Calcium imaging}

Individual flies tested the previous day in behavior experiments were anesthetized by cooling. The brain and ventral nerve cord were removed from the animal and placed into external saline composed of (in $\mathrm{mM}$ ) $103 \mathrm{NaCl}$, $3 \mathrm{KCl}, 5 \mathrm{~N}$-tris(hydroxymethyl) methyl-2-aminoethane-sulfonic acid, 10 trehalose dihydrate, 10 glucose, 26 NaHCO3, $1 \mathrm{NaH2PO} 4,4 \mathrm{MgCl}$, $3 \mathrm{KCl}, 2$ sucrose, and $1.5 \mathrm{CaCl} 2$ (280-290 mOsm, pH 7.3; components from Sigma Aldrich). The connective tissue was removed using fine forceps and the brain with attached ventral nerve cord was transferred to a chamber (Series 20 Chamber, Warner Instruments) superfused with external saline (carboxygenated with $95 \% \mathrm{O} 2 / 5 \% \mathrm{CO} 2$ ) and held into place via a custom holder.

The GCaMP6f signal was visualized in pIP10 via two-photon imaging with a laser (Chameleon, Coherent) tuned to 920 nm, a Zeiss Examiner Z1 with W Plan-Apochromat 20X/1.0 DIC M27 75 mm water immersion objective, and Zeiss Zen Software. The GCaMP signal was monitored in a single plane for 80 cycles at $391 \mathrm{~ms} / \mathrm{cycle}$ for a total imaging bout scan time of $\sim 30 \mathrm{~s}$. This $30 \mathrm{~s}$ imaging bout consisted of a $5 \mathrm{~s}$ baseline, activation of the CsChrimsontdTomato-expressing pC2I neurons via a constant-on, $5 \mathrm{~s} 635 \mathrm{~nm}$ LED pulse, and $20 \mathrm{~s}$ of post-pC2I-stimulation imaging. After a $30 \mathrm{~s}$ break, another imaging bout was run. The following $\mathrm{pC2}$-stimulating $635 \mathrm{~nm}$ light intensities were used from low to high: $0.2,0.5,0.9,1.3,1.9,2.3,3.7,5.3$, and $16.6 \mu \mathrm{W} / \mathrm{mm}^{2}$ (pE-4000, CoolLED) through the objective. This stimulation cycle was repeated three times and responses were averaged across trials. Because 8$10 \%$ of $635 \mathrm{~nm}$ light passes through the Drosophila cuticle (Inagaki et al., 2013; Ding et al., 2019), the 0.2 through $1.9 \mu \mathrm{W} / \mathrm{mm}^{2}$ irradiances approximately corresponded with the light stimuli given during behavior experiments. Data from the $1.9 \mu \mathrm{W} / \mathrm{mm}^{2}$ stimulus was used in Figure 5 and Supplemental Figure 4 because this light intensity 
corresponded to the light intensity used for optogenetic behavior analyses. A custom band-pass filter (Chroma) allowed constant visualization of the GCaMP6f signal while stimulating with $635 \mathrm{~nm}$ light.

Regions of interest (ROI) were manually drawn around pIP10 arbors. An additional background ROI was drawn to subtract stimulus noise from the raw GCaMP6f signal. After background subtraction the baseline data points were averaged to determine baseline fluorescence $\mathrm{FO}$, and the calcium signal was normalized to the baseline and multiplied by 100 ( $\triangle \mathrm{F} / \mathrm{FO} \%$ : Fx-FO/FO * 100). The average response during the 5 -second pC2I stimulation was used as the physiological connection value plotted in Figure $5 \mathrm{G}$ and Sup. Fig. 4D. Tissues were immediately fixed after GCaMP imaging and processed through immunohistochemistry, expanded, imaged, and analyzed to quantify anatomical connectivity.

\section{Irradiance calculations for behavior and calcium imaging experiments}

Irradiance was measured using a ThorLabs PM100D Compact Power and Energy Meter with a Console S130C Slim Photodiode Power Sensor. For behavior experiments, the sensor (diameter, $9.5 \mathrm{~mm}$ ) was positioned in the same location as the arena (diameter, $10.5 \mathrm{~mm}$ ) directly over the recording chamber microphone. Irradiance was calculated as the raw light power measured divided by the area of the sensor $\left(70.88 \mathrm{~mm}^{2}\right)$.

For calcium imaging experiments, the $635 \mathrm{~nm}$ LED stimulus (pE4000, CoolLED) was delivered (with stacked 2.0 and 1.0 neutral density filters in the beam path) through a Zeiss Examiner Z1 with W Plan-Apochromat 20X/1.0 DIC M27 $75 \mathrm{~mm}$ water immersion objective. The LED beam size was calculated using a beam profiler (WinCamDUCD12, DataRay) with the sensor placed at approximately the same distance from the objective as the sample during experiments $(2 \mathrm{~mm})$. This yielded a $1 / \mathrm{e}^{2}$ beam area of $0.95 \mathrm{~mm}^{2}$. Light power was also measured with the sensor placed $2 \mathrm{~mm}$ away from the center of the objective. In an effort to measure the light power of the focused beam and reduce the amount of unfocused or reflected light from being measured by the $70.88 \mathrm{~mm}^{2}$ sensor, a painted black foil sheath was placed over the sensor with an opening for the objective to deliver light. Irradiance was calculated as the raw light power measured divided by the $0.95 \mathrm{~mm}^{2}$ focused beam area.

\section{Figure preparation}

Images and videos were generated in VVD Viewer. Gamma, alpha, and saturation values were adjusted in VVD Viewer to facilitate visualization of overlapping structures. Images were then imported to and assembled in Adobe Illustrator. Scale bars of expanded samples are not adjusted to show pre-expansion size, and thus, show the size of structures after $8 \mathrm{X}$ expansion. Microscope objective and fly illustrations in Figures 1 and 5, and neural processes in Supplemental Figure 2 were created with BioRender.com.

\section{References}

Andlauer TFM, Scholz-Kornehl S, Tian R, Kirchner M, Babikir HA, Depner H, Loll B, Quentin C, Gupta VK, Holt MG, Dipt S, Cressy M, Wahl MC, Fiala A, Selbach M, Schwärzel M, Sigrist SJ (2014) Drep-2 is a novel synaptic protein important for learning and memory. Elife 3:1-24.

Arthur BJ, Ding Y, Sosale M, Khalif F, Kim E, Waddell P, Turaga SC, Stern DL (2021) SongExplorer: A deep learning workflow for discovery and segmentation of animal acoustic communication signals. bioRxiv:2021.03.26.437280.

Arthur BJ, Sunayama-Morita T, Coen P, Murthy M, Stern DL (2013) Multi-channel acoustic recording and automated analysis of Drosophila courtship songs. BMC Biol 11:11.

Auer TO, Khallaf MA, Silbering AF, Zappia G, Ellis K, Álvarez-Ocaña R, Arguello JR, Hansson BS, Jefferis GSXE, Caron SJC, Knaden M, Benton R (2020) Olfactory receptor and circuit evolution promote host 
specialization. Nature 579:402-408.

Bates AS, Schlegel P, Roberts RJV, Drummond N, Tamimi IFM, Turnbull R, Zhao X, Marin EC, Popovici PD, Dhawan S, Jamasb A, Javier A, Serratosa Capdevila L, Li F, Rubin GM, Waddell S, Bock DD, Costa M, Jefferis GSXE (2020) Complete Connectomic Reconstruction of Olfactory Projection Neurons in the Fly Brain. Curr Biol 30:3183-3199.e6.

Bourne JN, Harris KM (2012) Nanoscale analysis of structural synaptic plasticity. Curr Opin Neurobiol 22:372382.

Buhmann J, Sheridan A, Malin-Mayor C, Schlegel P, Gerhard S, Kazimiers T, Krause R, Nguyen TM, Heinrich L, Lee W-CA, Wilson R, Saalfeld S, Jefferis GSXE, Bock DD, Turaga SC, Cook M, Funke J (2021) Automatic detection of synaptic partners in a whole-brain Drosophila electron microscopy data set. Nat Methods 2021187 18:771-774.

Bumbarger DJ, Riebesell M, Rödelsperger C, Sommer RJ (2013) System-wide rewiring underlies behavioral differences in predatory and bacterial-feeding nematodes. Cell 152:109-119.

Calhoun AJ, Pillow JW, Murthy M (2019) Unsupervised identification of the internal states that shape natural behavior. Nat Neurosci 20192212 22:2040-2049.

Chen BC et al. (2014a) Lattice light-sheet microscopy: Imaging molecules to embryos at high spatiotemporal resolution. Science (80- ) 346.

Chen T-W, Wardill TJ, Sun Y, Pulver SR, Renninger SL, Baohan A, Schreiter ER, Kerr RA, Orger MB, Jayaraman V, Looger LL, Svoboda K, Kim DS (2013) Ultra-sensitive fluorescent proteins for imaging neuronal activity. Nature 499:295.

Chen Y, Akin O, Nern A, Tsui CK, Pecot MY, Zipursky SL (2014b) Cell-type Specific Labeling of Synapses in vivo through Synaptic Tagging with Recombination (STaR). Neuron 81:280.

Çiçek Ö, Abdulkadir A, Lienkamp SS, Brox T, Ronneberger O (2016) 3D U-Net: Learning Dense Volumetric Segmentation from Sparse Annotation. Lect Notes Comput Sci (including Subser Lect Notes Artif Intell Lect Notes Bioinformatics) 9901 LNCS:424-432.

Clemens J, Coen P, Roemschied FA, Pereira TD, Mazumder D, Aldarondo DE, Pacheco DA, Murthy M (2018) Discovery of a New Song Mode in Drosophila Reveals Hidden Structure in the Sensory and Neural Drivers of Behavior. Curr Biol 28:2400-2412.e6.

Consortium Mic et al. (2021) Functional connectomics spanning multiple areas of mouse visual cortex. bioRxiv:2021.07.28.454025.

Cook SJ, Jarrell TA, Brittin CA, Wang Y, Bloniarz AE, Yakovlev MA, Nguyen KCQ, Tang LTH, Bayer EA, Duerr JS, Bülow HE, Hobert O, Hall DH, Emmons SW (2019) Whole-animal connectomes of both Caenorhabditis elegans sexes. Nat 20195717763 571:63-71.

Deutsch D, Clemens J, Thiberge SY, Guan G, Murthy M (2019) Shared Song Detector Neurons in Drosophila Male and Female Brains Drive Sex-Specific Behaviors. Curr Biol 29:3200-3215.e5.

Di Tommaso P, Chatzou M, Floden EW, Barja PP, Palumbo E, Notredame C (2017) Nextflow enables reproducible computational workflows. Nat Biotechnol 35:316-319.

Ding Y, Lillvis JL, Cande J, Berman GJ, Arthur BJ, Long X, Xu M, Dickson BJ, Stern DL (2019) Neural Evolution of Context-Dependent Fly Song. Curr Biol 29:1089-1099.e7. 
Dionne H, Hibbard KL, Cavallaro A, Kao J-C, Rubin GM (2018) Genetic Reagents for Making Split-GAL4 Lines in Drosophila. Genetics 209:31-35.

Ehmann N, Owald D, Kittel RJ (2017) Drosophila active zones: From molecules to behaviour. Neurosci Res.

Eichler K, Li F, Litwin-Kumar A, Park Y, Andrade I, Schneider-Mizell CM, Saumweber T, Huser A, Eschbach C, Gerber B, Fetter RD, Truman JW, Priebe CE, Abbott LF, Thum AS, Zlatic M, Cardona A (2017) The complete connectome of a learning and memory centre in an insect brain. Nat 20175487666 548:175-182.

Feng K, Palfreyman MT, Häsemeyer M, Talsma A, Dickson BJ (2014) Ascending SAG Neurons Control Sexual Receptivity of Drosophila Females. Neuron 83:135-148.

Gao R et al. (2019) Cortical column and whole-brain imaging with molecular contrast and nanoscale resolution. Science (80-) 363.

Greenspan RJ, Ferveur J-F (2000) Courtship in Drosophila. Annu Rev Genet 34:205-232.

Güiza J, Barría I, Sáez JC, Vega JL (2018) Innexins: Expression, Regulation, and Functions. Front Physiol 0:1414.

Häsemeyer M, Yapici N, Heberlein U, Dickson BJ (2009) Sensory Neurons in the Drosophila Genital Tract Regulate Female Reproductive Behavior. Neuron 61:511-518.

Hildebrand DGC et al. (2017) Whole-brain serial-section electron microscopy in larval zebrafish. Nat 2017 5457654 545:345-349.

Huang GB, Scheffer LK, Plaza SM (2018) Fully-Automatic Synapse Prediction and Validation on a Large Data Set. Front Neural Circuits 12:87.

Inagaki HK, Jung Y, Hoopfer ED, Wong AM, Mishra N, Lin JY, Tsien RY, Anderson DJ (2013) Optogenetic control of Drosophila using a red-shifted channelrhodopsin reveals experience-dependent influences on courtship. Nat Methods 11:325-332.

Januszewski M, Kornfeld J, Li PH, Pope A, Blakely T, Lindsey L, Maitin-Shepard J, Tyka M, Denk W, Jain V (2018) High-precision automated reconstruction of neurons with flood-filling networks. Nat Methods 2018158 15:605-610.

Jefferis GSXE, Potter CJ, Chan AM, Marin EC, Rohlfing T, Maurer CR, Luo L (2007) Comprehensive maps of Drosophila higher olfactory centers: spatially segregated fruit and pheromone representation. Cell 128:1187-1203.

Jenett A et al. (2012) A GAL4-driver line resource for Drosophila neurobiology. Cell Rep 2:991-1001.

Klapoetke NC et al. (2014) Independent optical excitation of distinct neural populations. Nat Methods 11:338346.

Kohatsu S, Yamamoto D (2015) Visually induced initiation of Drosophila innate courtship-like following pursuit is mediated by central excitatory state. Nat Commun 6:1-9.

Kohl J, Ostrovsky AD, Frechter S, Jefferis GSXE (2013) A Bidirectional Circuit Switch Reroutes Pheromone Signals in Male and Female Brains. Cell 155:1610.

Kurtovic A, Widmer A, Dickson BJ (2007) A single class of olfactory neurons mediates behavioural responses to a Drosophila sex pheromone. Nat 20064467135 446:542-546.

Kurtzer GM, Sochat V, Bauer MW (2017) Singularity: Scientific containers for mobility of compute. PLoS One 
12:e0177459.

Lillvis JL (2021) JaneliaSciComp/exllsm-neuron-segmentation: Release 1.0.1.

Lillvis JL, Rokicki K, Goina C (2021) JaneliaSciComp/exllsm-circuit-reconstruction: Release 1.4.1.

Marder E (2011) Variability, compensation, and modulation in neurons and circuits. Proc Natl Acad Sci U S A 108 Suppl:15542-15548.

Marder E, Goeritz ML, Otopalik AG (2015) Robust circuit rhythms in small circuits arise from variable circuit components and mechanisms. Curr Opin Neurobiol 31:156-163.

Marin EC, Jefferis GSX., Komiyama T, Zhu H, Luo L (2002) Representation of the Glomerular Olfactory Map in the Drosophila Brain. Cell 109:243-255.

Markert SM, Britz S, Proppert S, Lang M, Witvliet D, Mulcahy B, Sauer M, Zhen M, Bessereau J-L, Stigloher C (2016) Filling the gap: adding super-resolution to array tomography for correlated ultrastructural and molecular identification of electrical synapses at the $\mathrm{C}$. elegans connectome.

https://doi.org/101117/1NPh34041802 3:041802.

Merkel D (2014) Docker: lightweight Linux containers for consistent development and deployment. Linux J 239.

Nagy JI, Pereda AE, Rash JE (2018) Electrical synapses in mammalian CNS: Past eras, present focus and future directions. Biochim Biophys Acta - Biomembr 1860:102-123.

Otsuna H, Ito M, Kawase T (2018) Color depth MIP mask search: a new tool to expedite Split-GAL4 creation. bioRxiv:318006.

Phelan P, Goulding LA, Tam JLY, Allen MJ, Dawber RJ, Davies JA, Bacon JP (2008) Molecular Mechanism of Rectification at Identified Electrical Synapses in the Drosophila Giant Fiber System. Curr Biol 18:1955-1960.

Roemschied FA, Pacheco DA, Ireland EC, Li X, Aragon MJ, Pang R, Murthy M (2021) F LEXIBLE CIRCUIT MECHANISMS FOR CONTEXT - DEPENDENT. :1-22.

Rokicki K, Kawase T (2021) JaneliaSciComp/exllsm-fiji-plugins: Release 1.1.1.

Rokicki K, Lillvis JL (2021) JaneliaSciComp/exllsm-synapse-detector: Release 1.4.1.

Ryan K, Lu Z, Meinertzhagen IA (2016) The CNS connectome of a tadpole larva of Ciona intestinalis (L.) highlights sidedness in the brain of a chordate sibling. Elife 5.

Scheffer LK et al. (2020) A connectome and analysis of the adult drosophila central brain. Elife 9:1-74.

Schindelin J, Arganda-Carreras I, Frise E, Kaynig V, Longair M, Pietzsch T, Preibisch S, Rueden C, Saalfeld S, Schmid B, Tinevez J-Y, White DJ, Hartenstein V, Eliceiri K, Tomancak P, Cardona A (2012) Fiji: an opensource platform for biological-image analysis. Nat Methods 9:676-682.

Schneider-Mizell CM, Gerhard S, Longair M, Kazimiers T, Li F, Zwart MF, Champion A, Midgley FM, Fetter RD, Saalfeld S, Cardona A (2016) Quantitative neuroanatomy for connectomics in Drosophila. Elife 5.

Seeholzer LF, Seppo M, Stern DL, Ruta V (2018) Evolution of a central neural circuit underlies Drosophila mate preferences. Nature 559:564-569.

Simonyan K, Zisserman A (2014) Very Deep Convolutional Networks for Large-Scale Image Recognition. 3rd Int Conf Learn Represent ICLR 2015 - Conf Track Proc. 
Sommer C, Straehle C, Kothe U, Hamprecht FA (2011) llastik: Interactive learning and segmentation toolkit. In: Eighth IEEE International Symposium on Biomedical Imaging (ISBI). Proceedings,, pp 230-233. IEEE.

Stern DL, Crocker J, Ding Y, Frankel N, Kappes G, Kim E, Kuzmickas R, Lemire A, Mast JD, Picard S (2017) Genetic and Transgenic Reagents for Drosophila simulans, D. mauritiana, D. yakuba, D. santomea, and D. virilis. G3 (Bethesda) 7:1339-1347.

Stockinger P, Kvitsiani D, Rotkopf S, Tirián L, Dickson BJ (2005) Neural circuitry that governs Drosophila male courtship behavior. Cell 121:795-807.

Syrjanen J, Michalski K, Kawate T, Furukawa H (2021) On the molecular nature of large-pore channels. J Mol Biol 433:166994.

Takemura S et al. (2013) A visual motion detection circuit suggested by Drosophila connectomics. Nature 500:175-181.

Takemura S et al. (2015) Synaptic circuits and their variations within different columns in the visual system of Drosophila. Proc Natl Acad Sci 112:13711-13716.

Takemura S et al. (2017) A connectome of a learning and memory center in the adult Drosophila brain. Elife 6.

Tanaka R, Higuchi T, Kohatsu S, Sato K, Yamamoto D (2017) Optogenetic activation of the fruitless-labeled circuitry in Drosophila subobscura males induces mating motor acts. J Neurosci 37:11662-11674.

Tillberg PW, Chen F, Piatkevich KD, Zhao Y, Yu C-C, English BP, Gao L, Martorell A, Suk H-J, Yoshida F, DeGennaro EM, Roossien DH, Gong G, Seneviratne U, Tannenbaum SR, Desimone R, Cai D, Boyden ES (2016) Proteinretention expansion microscopy of cells and tissues labeled using standard fluorescent proteins and antibodies. Nat Biotechnol 34:987-992.

Tirian L, Dickson B (2017) The VT GAL4, LexA, and split-GAL4 driver line collections for targeted expression in the Drosophila nervous system. bioRxiv:198648.

Tuthill JC, Nern A, Holtz SL, Rubin GM, Reiser MB (2013) Contributions of the 12 neuron classes in the fly lamina to motion vision. Neuron 79:128.

Vactor D Van, Sigrist SJ (2017) Presynaptic Morphogenesis, Active Zone Organization and Structural Plasticity in Drosophila. Curr Opin Neurobiol 43:119.

Valdes-Aleman J, Fetter RD, Sales EC, Heckman EL, Venkatasubramanian L, Doe CQ, Landgraf M, Cardona A, Zlatic M (2021) Comparative Connectomics Reveals How Partner Identity, Location, and Activity Specify Synaptic Connectivity in Drosophila. Neuron 109:105-122.e7.

von Philipsborn AC, Liu T, Yu JY, Masser C, Bidaye SS, Dickson BJ (2011) Neuronal control of Drosophila courtship song. Neuron 69:509-522.

Wagh DA, Rasse TM, Asan E, Hofbauer A, Schwenkert I, Dürrbeck H, Buchner S, Dabauvalle MC, Schmidt M, Qin G, Wichmann C, Kittel R, Sigrist SJ, Buchner E (2006) Bruchpilot, a protein with homology to ELKS/CAST, is required for structural integrity and function of synaptic active zones in Drosophila. Neuron 49:833-844.

Wan Y, Otsuna H, Chien C-B, Hansen C (2012) FluoRender: An Application of 2D Image Space Methods for 3D and 4D Confocal Microscopy Data Visualization in Neurobiology Research. IEEE Pacific Vis Symp:201-208.

Wang F, Wang K, Forknall N, Patrick C, Yang T, Parekh R, Bock D, Dickson BJ (2020a) Neural circuitry linking mating and egg laying in Drosophila females. Nat 20205797797 579:101-105. 
Wang K, Wang F, Forknall N, Yang T, Patrick C, Parekh R, Dickson BJ (2020b) Neural circuit mechanisms of sexual receptivity in Drosophila females. Nat 20205897843 589:577-581.

White J, Southgate E, Thomson J, Brenner S (1986) The structure of the nervous system of the nematode Caenorhabditis elegans. Philos Trans R Soc London B, Biol Sci 314:1-340.

Wong AM, Wang JW, Axel R (2002) Spatial Representation of the Glomerular Map in the Drosophila Protocerebrum. Cell 109:229-241.

Wu CL, Shih MFM, Lai JSY, Yang HT, Turner GC, Chen L, Chiang AS (2011) Heterotypic Gap Junctions between Two Neurons in the Drosophila Brain Are Critical for Memory. Curr Biol 21:848-854.

Yang C, Rumpf S, Xiang Y, Gordon MD, Song W, Jan LY, Jan Y-N (2009) Control of the Postmating Behavioral Switch in Drosophila Females by Internal Sensory Neurons. Neuron 61:519.

Zaharia M, Xin RS, Wendell P, Das T, Armbrust M, Dave A, Meng X, Rosen J, Venkataraman S, Franklin MJ, Ghodsi A, Gonzalez J, Shenker S, Stoica I (2016) Apache spark: A unified engine for big data processing. Commun ACM 59:56-65.

Zheng Z et al. (2018) A Complete Electron Microscopy Volume of the Brain of Adult Drosophila melanogaster. Cell 174:730-743.e22. 